\title{
Theoretical Issues
}

\author{
Marc Vanderhaeghen \\ Physics Department, The College of William \& Mary, Williamsburg, VA 23187, USA \\ Theory Center, Jefferson Lab, 12000 Jefferson Ave, Newport News, VA 23606, USA
}

\begin{abstract}
The theoretical issues in the interpretation of the precision measurements of the nucleon-to- $\Delta$ transition by means of electromagnetic probes are highlighted. The results of these measurements are confronted with the state-of-the-art calculations based on chiral effective-field theories (EFT), lattice $\mathrm{QCD}$, large- $N_{c}$ relations, perturbative $\mathrm{QCD}$, and QCD-inspired models. The link of the nucleon-to- $\Delta$ form factors to generalized parton distributions (GPDs) is also discussed.

Keywords: $\Delta(1232)$, electomagnetic form factors, pion production

PACS: $14.20 . \mathrm{Gk}, 13.40 . \mathrm{Gp}, 13.60 . \mathrm{Le}$
\end{abstract}

\section{INTRODUCTION}

Both the nucleon and the $\Delta(1232)$ resonance - the first excited state of the nucleon - can be considered as quantum states in the rich and complex spectrum of the quark-gluon system. It is well established that the underlying theory which should describe this spectrum is Quantum Chromodynamics (QCD). However, direct calculations in QCD of quantities such as the baryon spectrum, form factors, and parton distributions are extremely difficult, because they require non-perturbative methods. Only the lattice simulations have achieved some limited success to date in computing the hadron properties from first principles. Even then, such calculations are severely limited by the presently available computing power. To unravel the structure of the nucleon and understand the details of its spectrum requires therefore a dedicated effort on both experimental and theoretical sides.

The $\Delta$-resonance dominates many nuclear phenomena at energies above the pion-production threshold. In a laboratory, the $\Delta$ 's are produced in scattering the pion, photon, or electron beams off a nucleon target. High-precision measurements of the $N \rightarrow \Delta$ transition by means of electromagnetic probes became possible with the advent of the new generation of electron beam facilities, such as LEGS, BATES, ELSA, MAMI, and Jefferson Lab. Many such experimental programs devoted to the study of electromagnetic properties of the $\Delta$ have been completed in the past few years, see Refs. $[1,2,3,4]$ for reviews of the $\gamma N \Delta$ transition from both experimental and theoretical sides.

The electromagnetic $N \rightarrow \Delta$ (or, in short $\gamma N \Delta$ ) transition is predominantly of the magnetic dipole (M1) type. A first understanding of the $\gamma N \Delta$ transition can be obtained based on symmetries of Quantum Chromodynamics (QCD) and its large number-of-color $\left(N_{c}\right)$ limit. The spin-flavor global symmetry of QCD, is utilized by many quark models and is exactly realized in the large- $N_{c}$ limit. In the quark-model picture, the $N \rightarrow \Delta$ transition is described by a spin flip of a quark in the $s$-wave state, which in the $\gamma N \Delta$ case leads to the magnetic dipole $(M 1)$ type of transition. Any $d$-wave admixture in the nucleon or the $\Delta$ wave functions allows also for the electric $(E 2)$ and Coulomb $(C 2)$ quadrupole transitions. Therefore, by measuring the latter two transitions, one is able to assess the presence of the $d$-wave components and hence quantify to which extent the nucleon or the $\Delta$ wave function deviates from the spherical shape. In this way one can hope to understand to which extent these particles are "deformed".

The $d$-wave component of $\Delta$ 's wave function can be separately assessed by measuring the electric quadrupole moment of the $\Delta$. However, this would be extremely difficult because of the tiny lifetime of the $\Delta$. The small $d$-state probability of the $\Delta$ 's wave function also enters into the $\Delta$ magnetic dipole moment, which is being measured, but the extraction of a small number from such a quantity is also very complicated. The $\gamma N \Delta$ transition, on the other hand, was accurately measured in the pion photo- and electro-production reactions in the $\Delta$-resonance energy region. The $E 2$ and $C 2$ transitions were found to be relatively small but non-zero at moderate momentum-transfers $\left(Q^{2}\right)$, the ratios $R_{E M}=E 2 / M 1$ and $R_{S M}=C 2 / M 1$ are at the level of a few percent.

Because the $\Delta$ excitation energy is only around $300 \mathrm{MeV}$ and because the $\Delta$-resonance almost entirely decays into $\pi N$, pions are expected to play a prominent role in the $\Delta$ properties. Early calculations of the $\gamma N \Delta$ transition within chiral bag models revealed the importance of the pion cloud effects. Recall that pions are the Goldstone bosons of the spontaneously broken chiral symmetry of QCD. As the strength of the Goldstone boson interactions is proportional 

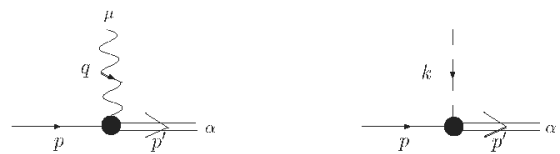

FIGURE 1. $\gamma^{*} N \Delta$ vertex (left panel) and $\pi N \Delta$ vertex (right panel). The four-momenta of the nucleon $(\Delta)$ and of the photon (pion) are given by $p\left(p^{\prime}\right)$ and $q(k)$ respectively. The four-vector index of the spin $3 / 2$ field is given by $\alpha$, and $\mu$ is the four-vector index of the photon field.

to their energy, at sufficiently low energy a perturbative expansion is possible. With the advent of the chiral effective field theory $(\chi \mathrm{EFT})$ of QCD $[5,6,7]$ and its extensions to the $\Delta$-resonance region, it has become possible to study the nucleon and $\Delta$-resonance properties in a profoundly different way. The advantages of such an approach are apparent: $\chi \mathrm{EFT}$ is a low-energy effective field theory of QCD and as such it provides a firm theoretical foundation, with all the relevant symmetries and scales of QCD built in consistently. The $\gamma N \Delta$ transition provides new challenges for $\chi \mathrm{EFT}$ as it involves the interplay of two light mass scales : the pion mass and the $N-\Delta$ mass difference.

Considerable progress has recently been achieved as well in the lattice QCD simulations of hadronic properties. They hold the promise to compute non-perturbative properties of QCD from first principles. The present state-of-theart results for hadronic structure quantities, such as the $\gamma N \Delta$ transition form factors, are obtained for pion masses above $300 \mathrm{MeV}$. Therefore, they can only be confronted with experiment after an extrapolation down to the physical pion mass of $140 \mathrm{MeV}$. Such extrapolation can be obtained with the aid of $\chi$ EFT, where the pion mass dependence is systematically calculable. The $\chi$ EFT framework thus bridges lattice QCD calculations and experiment.

The quark structure of the $N \rightarrow \Delta$ transition is accessible through the phenomenon of asymptotic freedom of QCD at short distances. In a hard scattering process, such as $\gamma^{*} N \rightarrow \gamma \Delta$ where the virtual photon $\gamma^{*}$ transfers a large momentum, the QCD factorization theorems allow one to separate the perturbative and non-perturbative stages of the interaction. In this way, one accesses in experiment the non-perturbative matrix elements parametrized in terms of new parton distributions, which are generalizations of the quark distributions from deep inelastic scattering experiments. One obtains in this way quark distribution information for the $N \rightarrow \Delta$ transition, and the $\gamma N \Delta$ form factors are obtained as first moments of such $N \rightarrow \Delta$ generalized parton distributions.

After introducing the definitions of $\gamma^{*} N \Delta$ form factors, the present status in our theoretical understanding of the $\gamma N \Delta$ transition is discussed.

\section{THE $\gamma^{*} N \Delta$ VERTEX AND FORM FACTORS}

The general spin structure of the pion- and photon-induced $N \rightarrow \Delta$ transitions, see Fig. 1, can be written as:

$$
\begin{aligned}
\bar{u}_{\alpha}\left(p^{\prime}\right) \Gamma_{\pi N \Delta}^{\alpha} u(p) & \equiv \frac{h_{A}}{2 f_{\pi}} \bar{u}_{\alpha}\left(p^{\prime}\right) k^{\alpha} T_{a}^{\dagger} u(p) F_{\pi N \Delta}\left(k^{2}\right) \\
\left\langle\Delta\left(p^{\prime}\right)\left|e J^{\mu}(0)\right| N(p)\right\rangle & \equiv i \sqrt{\frac{2}{3}} \frac{3 e\left(M_{\Delta}+M_{N}\right)}{2 M_{N}\left[\left(M_{\Delta}+M_{N}\right)^{2}+Q^{2}\right]} \\
\times \bar{u}_{\alpha}\left(p^{\prime}\right) & \left\{g_{M}\left(Q^{2}\right) \varepsilon^{\alpha \mu \rho \sigma} p_{\rho}^{\prime} q_{\sigma}+g_{E}\left(Q^{2}\right)\left(q^{\alpha} p^{\prime \mu}-q \cdot p^{\prime} \mathbf{g}^{\alpha \mu}\right) i \gamma_{5}+g_{C}\left(Q^{2}\right)\left(q^{\alpha} q^{\mu}-q^{2} g^{\alpha \mu}\right) i \gamma_{5}\right\} u(p)
\end{aligned}
$$

where $k$ is the pion and $q$ is the photon 4-momentum, $p\left(p^{\prime}\right)$ is the nucleon (the $\left.\Delta\right)$ 4-momentum, $M_{N}\left(M_{\Delta}\right)$ is the nucleon (the $\Delta$ ) mass, $u$ is the nucleon spinor, and $u_{c}$ represents the spin-3/2 $\Delta$ vector-spinor In Eqs. (1) and (2), the spin dependence in the $N$ and $\Delta$ spinors is understood. The operator $T_{a}^{+}$in Eq. (1), with $a=1,2,3$ corresponding with the Cartesian pion fields $\pi^{a}$, is the isospin $1 / 2 \rightarrow 3 / 2$ transition operator. The operator $J^{\mu}$ is the electromagnetic current operator, and the factor $\sqrt{2 / 3}$ in front of Eq. (2) corresponds with the isospin factor for the photon induced $p \rightarrow \Delta^{+}$ transition. Furthermore, $f_{\pi} \simeq 92.4 \mathrm{MeV}$ is the pion decay constant, $h_{A}$ is a dimensionless constant representing the strength of the $\pi N \rightarrow \Delta$ transition and thus related to the decay width of the $\Delta$ to $\pi N$. The strong transition form factor $F_{\pi N \Delta}$ is normalized as $F_{\pi N \Delta}\left(m_{\pi}^{2}\right)=1$. More details on definitions and conventions can be found in Ref. [4].

In Eq. (2), the electromagnetic form factors $g_{M}, g_{E}$, and $g_{C}$ represent the strength of the magnetic dipole, electric quadrupole, and Coulomb quadrupole $N \rightarrow \Delta$ transitions, respectively, as a function of the momentum transfer: 
$Q^{2}=-q^{2}$. Note that for the real photon case, $Q^{2}=0$, only the magnetic and electric transitions are possible, the Coulomb term drops out.

These electromagnetic form factors relate to the more conventional magnetic dipole $\left(G_{M}^{*}\right)$, electric quadrupole $\left(G_{E}^{*}\right)$ and Coulomb quadrupole $\left(G_{C}^{*}\right)$ form factors of Jones and Scadron [8] as follows :

$$
\begin{aligned}
G_{M}^{*}\left(Q^{2}\right) & =g_{M}+\frac{1}{Q_{+}^{2}}\left[\frac{1}{2}\left(-M_{\Delta}^{2}+M_{N}^{2}+Q^{2}\right) g_{E}+Q^{2} g_{C}\right] \\
G_{E}^{*}\left(Q^{2}\right) & =\frac{1}{Q_{+}^{2}}\left[\frac{1}{2}\left(-M_{\Delta}^{2}+M_{N}^{2}+Q^{2}\right) g_{E}+Q^{2} g_{C}\right] \\
G_{C}^{*}\left(Q^{2}\right) & =\frac{1}{Q_{+}^{2}}\left[\left(-M_{\Delta}^{2}+M_{N}^{2}+Q^{2}\right) g_{C}-2 M_{\Delta}^{2} g_{E}\right]
\end{aligned}
$$

where $Q_{ \pm}$is defined as: $Q_{ \pm} \equiv \sqrt{\left(M_{\Delta} \pm M_{N}\right)^{2}+Q^{2}}$.

Equivalently, one can also parametrize the $\gamma^{*} N \Delta$ transition through three helicity amplitudes $A_{1 / 2}, A_{3 / 2}$ and $S_{1 / 2}$, which are defined in the $\Delta$ rest frame through the following matrix elements of the electromagnetic current operator:

$$
\begin{aligned}
A_{3 / 2} & \equiv-\frac{e}{\sqrt{2 q_{\Delta}}} \frac{1}{\left(4 M_{N} M_{\Delta}\right)^{1 / 2}}\left\langle\Delta(\overrightarrow{0},+3 / 2)\left|\mathbf{J} \cdot \varepsilon_{\lambda=+1}\right| N(-\vec{q},+1 / 2)\right\rangle, \\
A_{1 / 2} & \equiv-\frac{e}{\sqrt{2 q_{\Delta}}} \frac{1}{\left(4 M_{N} M_{\Delta}\right)^{1 / 2}}\left\langle\Delta(\overrightarrow{0},+1 / 2)\left|\mathbf{J} \cdot \varepsilon_{\lambda=+1}\right| N(-\vec{q},-1 / 2)\right\rangle, \\
S_{1 / 2} & \equiv \frac{e}{\sqrt{2 q_{\Delta}}} \frac{1}{\left(4 M_{N} M_{\Delta}\right)^{1 / 2}}\left\langle\Delta(\overrightarrow{0},+1 / 2)\left|J^{0}\right| N(-\vec{q},+1 / 2)\right\rangle,
\end{aligned}
$$

where the spin projections are along the $z$-axis (chosen along the virtual photon direction) and where the transverse photon polarization vector entering in $A_{1 / 2}$ and $A_{3 / 2}$ is given by $\varepsilon_{\lambda=+1}=-1 / \sqrt{2}(1, i, 0)$. Furthermore in Eq. (5), $e$ is the proton electric charge, related to the fine-structure constant as $\alpha_{e m} \equiv e^{2} /(4 \pi) \simeq 1 / 137$, and $q_{\Delta}$ is the magnitude of the virtual photon three-momentum in the $\Delta$ rest frame:

$$
q_{\Delta} \equiv|\mathbf{q}|=\frac{Q_{+} Q_{-}}{2 M_{\Delta}}
$$

The helicity amplitudes are functions of the photon virtuality $Q^{2}$, and can be expressed in terms of the Jones-Scadron $\gamma^{*} N \Delta$ form factors, by using Eq. (2), as :

$$
A_{3 / 2}=-N \frac{\sqrt{3}}{2}\left\{G_{M}^{*}+G_{E}^{*}\right\}, \quad A_{1 / 2}=-N \frac{1}{2}\left\{G_{M}^{*}-3 G_{E}^{*}\right\}, \quad S_{1 / 2}=N \frac{q_{\Delta}}{\sqrt{2} M_{\Delta}} G_{C}^{*},
$$

where $N$ is defined as :

$$
N \equiv \frac{e}{2}\left(\frac{Q_{+} Q_{-}}{2 M_{N}^{3}}\right)^{1 / 2} \frac{\left(M_{N}+M_{\Delta}\right)}{Q_{+}} .
$$

The above helicity amplitudes are expressed in units $\mathrm{GeV}^{-1 / 2}$, and reduce at $Q^{2}=0$ to the photo-couplings quoted by the Particle Data Group [14].

Experimentally, the $\gamma^{*} N \Delta$ helicity amplitudes are extracted from the $M 1, E 2$, and $C 2$ multipoles for the $\gamma^{*} N \rightarrow \pi N$ process at the resonance position, i.e. for $\pi N c . m$. energy $W=M_{\Delta}$. These pion electroproduction multipoles are denoted by $M_{1+}^{(3 / 2)}, E_{1+}^{(3 / 2)}$, and $S_{1+}^{(3 / 2)}$ (following standard notation), where the subscript refers to the partial wave $l=1$ in the $\pi N$ system and "+" indicates that the total angular momentum is $J=l+1 / 2$, being $3 / 2$. The superscript (3/2) in the multipole notation refers to the total isospin $3 / 2$. In order to extract resonance properties, one is particularly interested in the imaginary parts of the resonant multipoles at the resonance position, and defines :

$$
\bar{M}_{1+}^{(3 / 2)}\left(Q^{2}\right) \equiv \sqrt{\frac{2}{3}} a_{\Delta} \operatorname{Im} M_{1+}^{(3 / 2)}\left(Q^{2}, W=M_{\Delta}\right)
$$


and similar relations define $\bar{E}_{1+}^{(3 / 2)}$ and $\bar{S}_{1+}^{(3 / 2)}$. The definition of $a_{\Delta}$, which depends on kinematical quantities and the $\Delta$ width, can be found in [4].

From the multipoles at the resonance position, one can then extract the Jones-Scadron $\gamma^{*} N \Delta$ form factors as :

$$
\bar{M}_{1+}^{(3 / 2)}=N G_{M}^{*}, \quad \bar{E}_{1+}^{(3 / 2)}=-N G_{E}^{*}, \quad \bar{S}_{1+}^{(3 / 2)}=-N \frac{q_{\Delta}}{2 M_{\Delta}} G_{C}^{*} .
$$

Of special interest are the multipole ratios at the position of the $\Delta$ resonance. The ratio of electric quadrupole $(E 2)$ over magnetic dipole $(M 1)$ is denoted by : $R_{E M}=E 2 / M 1$ (sometimes also denoted by $E M R$ ), whereas the ratio of Coulomb quadrupole ( $C 2$ ) over magnetic dipole is denoted by $R_{S M}=C 2 / M 1$ (sometimes also denoted by $C M R$ ):

$$
R_{E M} \equiv E M R \equiv \frac{\bar{E}_{1+}^{(3 / 2)}}{\bar{M}_{1+}^{(3 / 2)}}=\frac{A_{1 / 2}-\frac{1}{\sqrt{3}} A_{3 / 2}}{A_{1 / 2}+\sqrt{3} A_{3 / 2}}, \quad \quad R_{S M} \equiv C M R \equiv \frac{\bar{S}_{1+}^{(3 / 2)}}{\bar{M}_{1+}^{(3 / 2)}}=\frac{\sqrt{2} S_{1 / 2}}{A_{1 / 2}+\sqrt{3} A_{3 / 2}} .
$$

Using the relations of Eq. (9), these ratios can be expressed in terms of the Jones-Scadron form factors as:

$$
R_{E M}=-\frac{G_{E}^{*}}{G_{M}^{*}}, \quad R_{S M}=-\frac{Q_{+} Q_{-}}{4 M_{\Delta}^{2}} \frac{G_{C}^{*}}{G_{M}^{*}} .
$$

The $Q^{2}=0$ limit of these $\gamma^{*} N \Delta$ form factors encodes some interesting static quantities. From the value of $G_{M}^{*}$ at $Q^{2}=0$, one can extract the static $N \rightarrow \Delta$ transition magnetic moment $\mu_{N \rightarrow \Delta}$ as [9]:

$$
\mu_{N \rightarrow \Delta}=\sqrt{\frac{M_{\Delta}}{M_{N}}} G_{M}^{*}(0)
$$

which is expressed in nuclear magnetons $\mu_{N} \equiv e /\left(2 M_{N}\right)$. Furthermore, from the value of $G_{E}^{*}$ at $Q^{2}=0$, one can extract a static $N \rightarrow \Delta$ quadrupole transition moment $Q_{N \rightarrow \Delta}$ as [9]:

$$
Q_{N \rightarrow \Delta}=-6 \sqrt{\frac{M_{\Delta}}{M_{N}}} \frac{1}{M_{N} q_{\Delta}(0)} G_{E}^{*}(0)
$$

where $q_{\Delta}(0)$ is obtained from Eq. (5) for $Q^{2}=0$, as $q_{\Delta}(0)=\left(M_{\Delta}^{2}-M_{N}^{2}\right) / 2 M_{\Delta}$.

The $\gamma N \Delta$ transition can be studied both in nucleon Compton scattering $\gamma N \rightarrow \gamma N$ and in the pion photoproduction reaction $\gamma N \rightarrow \pi N$. The highest precision data on the $\gamma N \Delta M 1$ and $E 2$ amplitudes at the real photon point $\left(Q^{2}=0\right)$ have been obtained in pion photoproduction experiments on a proton target, using linearly polarized photons, both by the MAMI/A2 Collaboration [10,11] and the LEGS Collaboration [12,13]. As summary of these experiments, the Particle Data Group quotes as values [14] :

$$
\begin{aligned}
& A_{1 / 2}=-(135 \pm 6) \quad\left[10^{-3} \mathrm{GeV}^{-1 / 2}\right], \\
& A_{3 / 2}=-(250 \pm 8) \quad\left[10^{-3} \mathrm{GeV}^{-1 / 2}\right] \\
& R_{E M}=-(2.5 \pm 0.5) \%
\end{aligned}
$$

The experimental information on the $M 1 \gamma N \Delta$ transition can equivalently be expressed in terms of $G_{M}^{*}(0)$ using Eq. (9) or equivalently in terms of the transition magnetic moment $\mu_{p \rightarrow \Delta^{+}}$using Eq. (12). Using the experimental values, Ref. [15] extracted the values:

$$
G_{M}^{*}(0)=3.02 \pm 0.03, \quad \quad \mu_{p \rightarrow \Delta^{+}}=[3.46 \pm 0.03] \mu_{N} .
$$

The value for the $E 2 \gamma N \Delta$ transition can equivalently be expressed in terms of a quadrupole transition moment using Eq. (13). Using the experimental values, Ref. [9] extracted:

$$
Q_{p \rightarrow \Delta^{+}}=-(0.0846 \pm 0.0033) \mathrm{fm}^{2} .
$$




\section{QUARK MODELS AND PION-CLOUD MODELS}

\section{Constituent quark models}

In a quark model, the nucleon appears as the ground state of a quantum-mechanical three-quark system in a confining potential. In such a picture, the ground state baryons (composed of the light up (u), down $(d)$ and strange $(s)$ quark flavors) are described by $S U(6)$ spin-flavor wave functions, supplemented by an antisymmetric color wave function.

In the Isgur-Karl model [16], the constituent quarks move in a harmonic oscillator type confining potential. For the ground state baryons, the three constituent quarks are in the $1 s$ oscillator ground state, corresponding with the [56]-plet of $S U(6)$. The harmonic oscillator states can be represented by $\left|B^{2 S+1} L_{J}\right\rangle_{t}$, where $B$ stands for either $N$ or $\Delta$ states, $S$ specifies the spin, $L$ the orbital angular momentum $(L=S, P, D, \ldots$ in the common spectroscopic notation), and $J$ the total angular momentum of the three-quark state. Furthermore, $t(=S, M, A)$ refers to the symmetry type : symmetric $(S)$, mixed symmetric $(M)$ or anti-symmetric $(A)$ under exchange of the quarks in both the spin-flavor and space parts of the baryon wave function. In the Isgur-Karl model, the long-range confining potential is supplemented by an interquark force corresponding with one-gluon exchange. The one-gluon exchange leads to a color hyperfine interaction between quarks $i$ and $j$ of the form:

$$
H_{h y p e r f i n e}^{i j}=\frac{2}{3} \frac{\alpha_{s}}{m_{i} m_{j}}\left\{\frac{8 \pi}{3} \mathbf{S}_{i} \cdot \mathbf{S}_{j} \delta^{3}\left(\mathbf{r}_{i j}\right)+\frac{1}{r_{i j}^{3}}\left[\frac{3\left(\mathbf{S}_{i} \cdot \mathbf{r}_{i j}\right)\left(\mathbf{S}_{j} \cdot \mathbf{r}_{i j}\right)}{r_{i j}^{2}}-\mathbf{S}_{i} \cdot \mathbf{S}_{j}\right]\right\},
$$

with $\alpha_{S}$ the strong coupling constant, $\mathbf{S}_{\mathbf{i}}\left(m_{i}\right)$ the spin (mass) of quark $i$, and where $\mathbf{r}_{\mathrm{ij}}\left(r_{i j}\right)$ specify the vector (distance) between quarks $i$ and $j$. The first term in Eq. (17) corresponds with a zero-range spin-spin interaction, whereas the second term corresponds with a tensor force. The color hyperfine interaction of Eq. (17) breaks the $S U(6)$ symmetry and leads to a mass splitting between $N(939)$ and $\Delta(1232)$, often referred to as the hyperfine splitting. It was found that it also predicts well the mass splittings between octet and decuplet baryons [17]. Furthermore, the tensor force in Eq. (17) will produce a $D$-state $(L=2)$ admixture in the $N$ and $\Delta$ ground states $[18,19]$. Because of this hyperfine interaction, the $N(939)$ and $\Delta(1232)$ states are described as superpositions of $S U(6)$ configurations. Including configurations up to the $2 \bar{h} \omega$ oscillator shell, they are given by (using the abovementioned spectroscopic notation):

$$
\begin{aligned}
|N(939)\rangle & =a_{S}\left|N^{2} S_{1 / 2}\right\rangle_{S}+a_{S}^{\prime}\left|N^{2} S_{1 / 2}^{\prime}\right\rangle_{S}+a_{M}\left|N^{2} S_{1 / 2}\right\rangle_{M}+a_{D}\left|N^{4} D_{1 / 2}\right\rangle_{M}, \\
|\Delta(1232)\rangle & =b_{S}\left|\Delta^{4} S_{3 / 2}\right\rangle_{S}+b_{S}^{\prime}\left|\Delta^{4} S_{3 / 2}^{\prime}\right\rangle_{S}+b_{D}\left|\Delta^{4} D_{3 / 2}\right\rangle_{S}+b_{D}^{\prime}\left|\Delta^{2} D_{3 / 2}\right\rangle_{M} .
\end{aligned}
$$

By diagonalizing the hyperfine interaction and fitting the results to the baryon spectrum, Isgur et al. [19] obtained the following values for the wave-function coefficients:

$$
\begin{array}{ll}
a_{S} \simeq 0.93, & a_{S}^{\prime} \simeq-0.29, \quad a_{M} \simeq-0.23, \quad a_{D} \simeq-0.04, \\
b_{S} \simeq 0.97, & b_{S}^{\prime} \simeq+0.20, \quad b_{D} \simeq-0.10, \quad b_{D}^{\prime} \simeq 0.07 .
\end{array}
$$

From these values it is evident that the $S$-wave component dominates the $N$ and $\Delta$ wave functions in a constituent quark model. The $\Delta(1232)$ resonance is obtained from the nucleon by a spin flip of one of the quarks in the $1 s$ state so as to give a state of total spin 3/2. Therefore the electromagnetic $N \rightarrow \Delta$ transition is dominantly a $M 1$ transition [20], see Fig. 2 (left panel). Using $S U(6)$ spin-flavor symmetry, i.e., setting $a_{S}=b_{S}=1$ and $a_{S}^{\prime}=a_{M}=a_{D}=b_{S}^{\prime}=b_{D}=b_{D}^{\prime}=0$ in Eqs. (18) and (19), yields the relation between the magnetic moments of proton and $p \rightarrow \Delta^{+}$as:

$$
\mu_{p \rightarrow \Delta^{+}}=\frac{2 \sqrt{2}}{3} \mu_{p}=2.63 \mu_{N} \quad(\mathrm{SU}(6) \text {-symmetric quark model }) .
$$

This is about $25 \%$ lower than the experimental number Eq. (15). $S U(6)$ breaking effects, due to the hyperfine interaction, decrease the quark model prediction even further to a value around $2.3 \mu_{N}$. A very similar small number is also obtained in the MIT bag model calculation of Ref. [21]. The underestimate of $\mu_{N \rightarrow \Delta}$ in quark models is hinting at the importance of degrees of freedom beyond constituents quarks as will be discussed further on.

The small non-zero values of $b_{D}$ and $b_{D}^{\prime}$ in Eq. (21) imply that the $D$-wave probability in the $\Delta(1232)$ ground state is around $1 \%$. As a result of such $D$-wave components, the $N$ and $\Delta$ charge densities become non-spherical. For a static charge distribution, a measure of the non-sphericity (or deformation) is given by its quadrupole moment. Since 

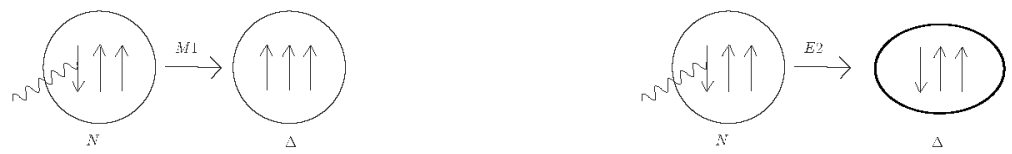

FIGURE 2. Schematic picture within a quark model of a $M 1$ (left panel) and $E 2$ (right panel) $N \rightarrow \Delta$ transition induced by the interaction of a photon with a single quark in the nucleon. The $M 1$ transition involves a $S$-wave spatial wave function, whereas the $E 2$ transition in this picture requires $N$ and/or $\Delta$ wave functions to have a $D$-wave component (indicated by a non-spherical shape).

the nucleon has spin 1/2, any intrinsic quadrupole moment of the nucleon cannot be directly measured though because angular momentum conservation forbids a non-zero matrix element of a ( $L=2)$ quadrupole operator between spin $1 / 2$ states. However this quadrupole deformation may reveal itself in an electromagnetically induced transition from the spin $1 / 2 N$ to the spin $3 / 2 \Delta$ state, see Fig. 2 (right panel). In this way, the tensor force between quarks gives rise to non-zero values for the $E 2 / M 1\left(R_{E M}\right)$ and $C 2 / M 1\left(R_{S M}\right)$ ratios. ${ }^{1}$

Isgur et al. [19], having obtained the $D$-wave admixture reflected in Eqs. (18)-(21), find $R_{E M} \simeq-0.41 \%$. Similar small negative values, in the range $-0.8 \%<R_{E M}<-0.3 \%$, were found in other non-relativistic quark model calculations $[23,24,25]$. Such a small value for $R_{E M}$ already indicates that any effect of deformation in the nucleon and/or $\Delta$ ground state is rather small and very sensitive to the details of the wave function.

Drechsel and Giannini [26] demonstrated that a calculation of the $N \rightarrow \Delta$ quadrupole transition within the quark model can be very sensitive to a truncation in the quark model basis. Due to such truncation (e.g. to a $2 \hbar \omega$ oscillator basis as in [19]) the charge-current operator for the quadrupole $N \rightarrow \Delta$ transition violates gauge invariance which can lead to $R_{E M}$ ratios which differ by an order of magnitude when calculated either from the charge or current operator. Drechsel and Giannini showed that when performing a $2 \hbar \omega$ truncation in the quark model basis, a calculation based on the charge operator is more stable than a calculation based on the current operator. By using $N$ and $\Delta 3$-quark wave functions which have a $D$-state probability of around $1 \%$, they obtained a value of $R_{E M} \simeq-2 \%$ and a $\Delta$ quadrupole moment $Q_{\Delta} \simeq-0.078 \mathrm{fm}^{2}[26,27]$.

The error induced due to the truncation in the quark model basis has been further investigated in the relativized quark model calculation of Capstick and Karl $[28,29]$. Using wave functions which have been expanded in a large harmonic oscillator basis up to $6 \bar{\hbar} \omega$ states, and are solutions of a relativized Hamiltonian, they obtained an even smaller negative value: $R_{E M} \simeq-0.21 \%$.

\section{Pion cloud models}

Even though the constituent quark model, despite its simplicity, is relatively successful in predicting the structure and spectrum of low-lying baryons, it under-predicts $\mu_{N \rightarrow \Delta}$ by more than $25 \%$ and leads to values for the $R_{E M}$ ratio which are typically smaller than experiment. More generally, constituent quark models do not satisfy the symmetry properties of the QCD Lagrangian. In nature, the up and down (current) quarks are nearly massless. In the exact massless limit, the QCD Lagrangian is invariant under $S U(2)_{L} \times S U(2)_{R}$ rotations of left $(L)$ and right $(R)$ handed quarks in flavor space. This chiral symmetry is spontaneously broken in nature leading to the appearance of massless Goldstone modes. For two flavors, there are three Goldstone bosons - pions, which acquire a mass due to the explicit breaking of chiral symmetry by the current quark masses. Since pions are the lightest hadrons, they dominate the long-distance behavior of hadron wave functions and yield characteristic signatures in the low-momentum transfer behavior of hadronic form factors. Furthermore, as the $\Delta(1232)$ resonance nearly entirely decays into $\pi N$ pions are of particular relevance to the electromagnetic $N \rightarrow \Delta$ transition. Therefore, a natural way to qualitatively improve on the above-mentioned constituent quark models is to include the pionic degrees of freedom.

An early investigation of the $\gamma N \Delta$ transition including pionic effects was performed by Kaelbermann and Eisenberg [30] within the chiral bag model. The chiral (or, cloudy) bag model improves the MIT bag model by introducing an elementary, perturbative pion which couples to quarks in the bag in such a way that chiral symmetry is restored [31].

\footnotetext{
1 The relation between the tensor force, $D$-wave admixture, and the electromagnetic $N \rightarrow \Delta$ transition was already pointed out in the early paper of Glashow [22].
} 
Bermuth et al. [32] partially corrected and repeated the calculation of Ref. [30] for two formulations of the chiral bag model: one with pseudoscalar (PS) surface coupling and one with pseudovector (PV) volume coupling of the pions. Lu et al. [33] in a later calculation within the chiral bag model improved upon the previous calculations by applying a correction for the center-of-mass motion of the bag. It was found that with a smaller bag radius, $R \approx 0.8$ $\mathrm{fm}$, one is able to obtain a reasonable size for the magnitude of the helicity amplitudes, see Table 1 . For such a small bag radius, the pionic effects are crucial as they account for around $75 \%$ of the total strength of the amplitude $A_{3 / 2}$.

For the $R_{E M}$ ratio, both Bermuth et al. and Lu et al. found severe cancellations between the $A_{1 / 2}$ and $A_{3 / 2}$ amplitudes in Eq. (10), leading to a very small value for $R_{E M}$, despite the 'reasonable' values for the helicity amplitudes, see Table 1. Bermuth et al. [32] therefore calculated $R_{E M}$ in the Siegert limit $(|\mathbf{q}| \rightarrow 0)$ where it can be obtained from $R_{S M}$ using the charge operator, and obtained the value $R_{E M} \simeq-2 \%$, close to experiment.

The $R_{E M}$ ratio has also been calculated in Skyrme models $[35,36,37]$, which have only pionic degrees of freedom. In the Skyrme model, the nucleon appears as a soliton solution of an effective nonlinear meson field theory. Wirzba and Weise [35] performed a modified Skyrme model calculation, at leading order in the number of colors $N_{c}$, based on the chiral effective Lagrangian, which corresponds with the standard Skyrme model supplemented by stabilizing fourth and sixth order terms in the pion fields. This calculation obtained $R_{E M}$ values between $-2.5 \%$ and $-6 \%$, depending on the coupling parameters of the stabilizing terms [35]. Certainly, the sign and order of magnitude of these results are consistent with the empirical result. Walliser and Holzwarth [37] included rotational corrections, which are of order $1 / N_{c}$, and lead to a quadrupole distortion of the classical soliton solution. Including such corrections, one finds a very good description of the photo-coupling s (see Table 1) and obtains a ratio $R_{E M}=-2.3 \%$, consistent with experiment.

A model which has both quark and pion degrees of freedom and interpolates between a constituent quark model and the Skyrme model is the chiral quark soliton model ( $\chi \mathrm{QSM}$ ). This model is based on the interaction of quarks with Goldstone bosons resulting from the spontaneous breaking of chiral symmetry. As for the Skyrme model, the $\chi \mathrm{QSM}$ is essentially based on the $1 / N_{c}$ expansion. Its effective chiral action has been derived from the instanton model of the QCD vacuum [38], which provides a natural mechanism of chiral symmetry breaking and enables one to generate dynamically the constituent quark mass. Although in reality the number of colors $N_{c}=3$, the extreme limit of large $N_{c}$ is known to yield useful insights. At large $N_{c}$ the nucleon is heavy and can be viewed as $N_{c}$ "valence" quarks bound by a self-consistent pion field (the "soliton") whose energy coincides with the aggregate energy of the quarks of the negative-energy Dirac continuum [39]. A successful description of static properties of baryons, such as mass splittings, axial constants, magnetic moments, form factors, has been achieved (typically at the $30 \%$ level or better). After reproducing masses and decay constants in the mesonic sector, the only free parameter left to be fixed in the baryonic sector is the constituent quark mass The good agreement of the $\chi$ QSM with the empirical situation is achieved for quark mass $M_{q} \simeq 420 \mathrm{MeV}$.

The $\chi$ QSM was also applied to the calculation of the $\gamma N \Delta$ transition $[40,41]$. In this model, the $\Delta$ is a bound state which corresponds to a soliton rotating in flavor space. The rotational energy is responsible for the $N-\Delta$ mass splitting. With a constituent quark mass $M_{q}=420 \mathrm{MeV}$, the empirical $N-\Delta$ mass splitting is well reproduced. The $\gamma N \Delta$ amplitudes are obtained taking rotational $\left(1 / N_{c}\right)$ corrections into account. In this way, Silva et al. [41] obtained as result: $R_{E M}=-2.1 \%$ (in the two-flavor case), fairly close to the experimental ratio, considering that in the $\chi \mathrm{QSM}$ calculation no parametrization adjustment has been made to the $N \rightarrow \Delta$ transition. However, the value of the $M 1 \gamma N \Delta$ amplitude is largely underpredicted in the $\chi$ QSM, which is also reflected in an underprediction of the magnitude of the photo-couplings, see Table 1.

The above calculations incorporating the chiral symmetry of QCD (to lowest order in the pion fields) highlight the role of the pionic degrees of freedom in the $\gamma N \Delta$ transition. A number of subsequent works have therefore revisited quark models, which break chiral symmetry, by including two-body exchange currents. As an example of such approach we consider the work of Buchmann, Hernandez and Faessler [42]. When the one-gluon exchange potential between quarks is complemented by a one-pion and one-sigma exchange potential between the quarks, the pion exchange gives rise to an additional tensor interaction between the quarks. The one-pion exchange then requires the presence of two-body exchange currents between the quarks due to current conservation. Within the non-relativistic framework, Buchmann et al. [42] found that the overall effect of the exchange currents on the dominant $M 1 \gamma N \Delta$ transition are relatively small due to cancellation. As a result, the transition magnetic moment comes out to be underpredicted: $\mu_{p \rightarrow \Delta^{+}} \simeq 2.5\left[\mu_{N}\right]$, similar to the non-relativistic quark model without exchange currents. This is also reflected in the helicity amplitudes obtained in [42] and quoted in Table 1 . They are smaller in the magnitude than the experimental values.

However, the two-body exchange currents lead to non-vanishing $\gamma N \Delta$ quadrupole ( $E 2$ or $C 2$ ) amplitudes [42], even if the quark wave functions have no $D$-state admixture. In this picture, the $\Delta$ is excited by flipping the spins of two quarks, see Fig. 3. According to Buchmann et al., this mechanism yields $R_{E M} \simeq-3.5 \%$. Within such a quark model, 


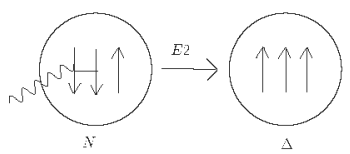

FIGURE 3. Schematic picture within a quark model of a $E 2 N \rightarrow \Delta$ transition induced by the interaction of a photon with two quarks in the nucleon (correlated through, e.g., one-pion exchange). Both $N$ and $\Delta$ wave functions are $S$-states.

Buchmann et al. also related the $N \rightarrow \Delta$ and $\Delta^{+}$quadrupole moments to the neutron charge radius, as :

$$
Q_{p \rightarrow \Delta^{+}}=\frac{1}{\sqrt{2}} r_{n}^{2}, \quad Q_{\Delta^{+}}=r_{n}^{2},
$$

see Ref. [43] for a discussion.

As a summary, Table 1 lists the $\gamma N \Delta$ photo-couplings $A_{1 / 2}$ and $A_{3 / 2}$ as well as the ratio $R_{E M}$ obtained in various model calculations.

TABLE 1. Summary of the values of the $\gamma N \Delta$ helicity amplitudes $A_{1 / 2}$ and $A_{3 / 2}$ at $Q^{2}=0$, and the ratio $R_{E M}\left(Q^{2}=0\right)$ in different models compared with experiment.

\begin{tabular}{|c|c|c|c|}
\hline & $A_{1 / 2}\left[10^{-3} \mathrm{GeV}^{-1 / 2}\right]$ & $A_{3 / 2}\left[10^{-3} \mathrm{GeV}^{-1 / 2}\right]$ & $R_{E M}[\%]$ \\
\hline $\begin{array}{l}\text { experiment } \\
\text { Ref. [14] }\end{array}$ & $-135 \pm 6$ & $-250 \pm 8$ & $-2.5 \pm 0.5$ \\
\hline SU(6) symmetry & -107 & -185 & 0 \\
\hline $\begin{array}{l}\text { non-relativistic quark models } \\
\text { Refs. }[18,19,23,24,25,26]\end{array}$ & -103 & -179 & -2 to 0 \\
\hline $\begin{array}{c}\text { relativized quark model } \\
\text { Refs. }[28,29]\end{array}$ & -108 & -186 & -0.2 \\
\hline MIIT bag model, Ref. [21] & -102 & -176 & 0 \\
\hline $\begin{array}{c}\text { chiral (cloudy) bag models } \\
\text { Ref. [32]: (PS, } \mathrm{R}=1 \mathrm{fm}) \\
\text { Ref. }[32]:(\mathrm{PV}, \mathrm{R}=1 \mathrm{fm}) \\
\text { Ref. }[33]:(+ \text { recoil }, \mathrm{R}=0.8 \mathrm{fm})\end{array}$ & $\begin{array}{l}-106 \\
-91 \\
-134\end{array}$ & $\begin{array}{l}-198 \\
-171 \\
-233\end{array}$ & $\begin{array}{l}-1.8 \\
-2.0 \\
-0.03\end{array}$ \\
\hline linear $\sigma$-model, Ref. [34] & -107 & -199 & -1.8 \\
\hline chromodielectric model, Ref. [34] & -70 & -131 & -1.9 \\
\hline $\begin{array}{c}\text { Skyrme models } \\
\text { Ref. [35] } \\
\text { Ref. [37]: }\left(+1 / N_{c} \text { corrections }\right)\end{array}$ & -136 & -259 & $\begin{array}{c}-6 \text { to }-2.5 \\
-2.3\end{array}$ \\
\hline $\begin{array}{l}\text { chiral quark soliton model } \\
\text { Ref. [41] : SU(2) flavor }\end{array}$ & -70.5 & -133 & -2.1 \\
\hline $\begin{array}{l}\text { quark model }+\pi, \sigma \text { exchange } \\
\text { Ref. [42] }\end{array}$ & -91 & -182 & -3.5 \\
\hline $\begin{array}{c}\text { chiral quark model }\left(\Lambda_{B}=0.8 \mathrm{GeV}\right) \\
\text { Ref. }[46]\end{array}$ & -124.3 & -244.7 & -3.1 \\
\hline
\end{tabular}

\section{Intrinsic quadrupole moment and the issue of shape}

Relations such as Eq. (23) were further interpreted by Buchmann and Henley [44] in terms of an intrinsic quadrupole moment of the nucleon and $\Delta$ states. The intrinsic quadrupole moment $Q^{0}$ of a static charge distribution $\rho(\vec{r})$ is given 


$$
Q^{0}=\int d^{3} \vec{r} \rho(\vec{r})\left(3 z^{2}-r^{2}\right),
$$

which is defined w.r.t. the body fixed frame. A charge distribution concentrated along the $z$-axis (symmetry axis of the system) corresponds with $Q^{0}>0$ (prolate deformation), whereas a charge distribution concentrated in the equatorial $x y$-plane corresponds with $Q^{0}<0$ (oblate deformation). This intrinsic quadrupole moment has to be distinguished from a measured (or spectroscopic) quadrupole moment $Q$. As an example, for a rigid rotor (which was considered within the context of the collective nuclear shell model [45]) these quantities are related as:

$$
Q=\frac{3 J_{z}^{2}-J(J+1)}{(J+1)(2 J+3)} Q^{0},
$$

where $J_{z}$ is the projection of the nucleon total spin $J$ onto its symmetry axis ( $z$-axis in a body fixed frame). The difference between $Q^{0}$ and $Q$ represents the averaging of the nonspherical charge distribution due to its rotational motion as seen in the laboratory frame. One verifies from Eq. (25) that the multiplication factor is zero for a spin $1 / 2$ particle, yiclding $Q_{p}=0$ for the proton. Eq. (25) does not preclude however that the proton has an intrinsic quadrupole moment $Q_{p}^{0}$.

Within a model where the nucleon consists of a spherically symmetric quark core surrounded by a pion with orbital angular momentum $l=1$, Buchmann and Henley obtain [44]:

$$
Q_{\Delta^{+}}^{0}=r_{n}^{2}=-Q_{p}^{0}
$$

Using the experimental neutron charge radius, this tells us that, the proton and $\Delta^{+}$have respectively a prolate and an oblate intrinsic deformation. In this hybrid (quark/pion-cloud) model, the pion cloud is fully responsible for the non-zero values of the quadrupole moments $Q_{p}^{0}$ and $Q_{\Delta^{+}}^{0}$, and hence for the non-spherical shape of these particles.

Estimates such as Eq. (26) of intrinsic quadrupole moments are surely useful to reveal details of a given model calculation and gain physical insight. One should keep in mind, however, that, even though a specific model such as the rigid rotor relates the intrinsic and total quadrupole moments, only the latter is directly related to observables.

\section{LARGE $N_{c}$ LIMIT}

Though the results from the different QCD inspired models discussed above may provide us with physical insight on the $\gamma N \Delta$ transition, they are not a rigorous consequence of QCD. In the following, we will discuss what is known on the $\gamma N \Delta$ transition from approaches which are a direct consequence of QCD in some limit, such as chiral effective field theory (chiral limit of small pion masses or momentum transfers) or lattice QCD simulations (continuum limit). Another first-principle technique is the $1 / N_{c}$ expansion of QCD proposed by 't Hooft [47] and furthermore developed by Witten [48]. The large- $N_{c}$ limit is unique in that it provides a perturbative parameter at all energy scales. This expansion has proved quite useful in describing the properties of baryons, such as, ground-state and excited baryon masses, magnetic moments, electromagnetic decays, see e.g., Refs. $[49,50]$ for reviews.

In the large $N_{c}$ limit, the baryons are infinitely heavy and can be treated as static. In this limit the baryon sector of QCD has an exact contracted $S U\left(2 N_{f}\right)$ spin-flavor symmetry, where $N_{f}$ is the number of light quark flavors. The large $N_{c}$ limit thus validates many of the quark model $S U(6)$ spin-flavor symmetry results without making any model assumption (such as assuming non-relativistic quark dynamics for the baryon wave functions). For example, the ratio of proton to neutron magnetic moments in the large $-N_{c}$ limit is predicted to be given by $\mu_{p} / \mu_{n}=-3 / 2$ in agreement with the naive quark model. Likewise at leading order in $1 / N_{c}$, the $N \rightarrow \Delta$ transition magnetic moment $\mu_{N \Delta}$ is related to the isovector combination of proton and neutron magnetic moments as [51]:

$$
\mu_{p \rightarrow \Delta^{+}}=\frac{1}{\sqrt{2}}\left(\mu_{p}-\mu_{n}\right)
$$

up to a correction of order $1 / N_{c}^{2}$. Using the empirical values for $\mu_{p}$ and $\mu_{n}$, one obtains in the large $N_{c}$ limit $\mu_{N \rightarrow \Delta}=3.23 \mu_{N}$, within $10 \%$ of the experimental value of Eq. (15).

The $R_{E M}$ ratio for the $\gamma N \Delta$ transition is shown to be of order $1 / N_{c}^{2}[52]$. Thus, the smallness of the $\gamma N \Delta R_{E M}$ ratio 
is naturally explained in the large $N_{c}$ limit. Using Eq. (10), this can equivalently be expressed as a large $N_{c}$ prediction for the $\gamma N \Delta$ helicity amplitudes:

$$
\frac{A_{3 / 2}}{A_{1 / 2}}=\sqrt{3}+\mathscr{Q}\left(\frac{1}{N_{c}^{2}}\right) .
$$

The large $N_{c}$ limit also allows to obtain relations between the $\Delta$ and $N \rightarrow \Delta$ quadrupole moments [53]:

$$
\frac{Q_{\Delta^{+}}}{Q_{p \rightarrow \Delta^{+}}}=\frac{2 \sqrt{2}}{5}+\mathscr{O}\left(\frac{1}{N_{c}^{2}}\right) .
$$

This result for $Q_{\Delta^{+}}$is different from the quark model ratio of Eq. (23) as explained in [53]. Using the phenomenological value of Eq. (16) for $Q_{p \rightarrow \Delta^{+}}$, the large $N_{c}$ relation of Eq. (29) yields for the $\Delta^{+}$quadrupole moment:

$$
Q_{\Delta^{+}}=-(0.048 \pm 0.002) \mathrm{fm}^{2}
$$

accurate up to corrections of order $1 / N_{c}^{2}$.

Buchmann, Hester and Lebed [53] derived recently another relation by making the additional assumption that the baryon charge radii and quadrupole moments arise from the same contributions. This assumption holds true when, e.g., the interaction between quarks arises from one-gluon or one-pion exchange, for which the ratio of spin-spin and tensor terms in the Hamiltonian is fixed. Under this assumption, one finds a large- $N_{c}$ relation between the $N \rightarrow \Delta$ quadrupole moment and the neutron charge radius $r_{n}^{2}[53]$ :

$$
Q_{p \rightarrow \Delta^{+}}=\frac{1}{\sqrt{2}} r_{n}^{2} \frac{N_{c}}{N_{c}+3} \sqrt{\frac{N_{c}+5}{N_{c}-1}},
$$

where both $Q_{p \rightarrow \Delta^{+}}$and $r_{n}^{2}$ are of order $1 / N_{c}^{2}$.

The factor on the $r h s$ of Eq. (31) after $r_{n}^{2}$ is unity both for the cases $N_{c}=3$ and $N_{c} \rightarrow \infty$, and deviates from unity by only about $1 \%$ for all values in between. Therefore the large $N_{c}$ limit predicts to good approximation the same relation of Eq. (23), $Q_{p \rightarrow \Delta^{+}}=1 / \sqrt{2} r_{n}^{2}$, which was also derived in the quark model and pion cloud model, and which was found to be consistent with the empirical value of Eq. (16) for $Q_{p \rightarrow \Delta^{+}}$.

More recently, Cohen and Lebed $[54,55]$ have argued that the relations, such as in Eqs. (29) and (31), may be significantly altered in the real world where the pions are light. When taking $N_{c} \rightarrow \infty$ at a fixed value of $m_{\pi}$, the $N-\Delta$ mass difference, which goes as $1 / N_{c}$, vanishes. Therefore, the "usual" large- $N_{c}$ limit implies that one is in the region $M_{\Delta}-M_{N} \ll m_{\pi}$, where, for instance, the $\Delta$ is stable. Cohen points out [54] that the large- $N_{c}$ limit and the chiral limit $\left(m_{\pi} \rightarrow 0\right)$ do not commute and for quantities which diverge in the chiral limit, such as the charge radii, one expects chiral corrections to dominate over the large- $N_{c}$ predictions. Although the region $M_{\Delta}-M_{N} \ll m_{\pi}$ is not accessible in nature, lattice QCD results, which are currently obtained in this regime, may provide an interesting testing ground for the above large $N_{c}$ predictions.

Finally, using an effective chiral Lagrangian, it was derived in Ref. [4], that in the large $N_{c}$ limit :

$$
R_{S M}=R_{E M}, \text { for } Q^{2}=0 \text { and } N_{c} \rightarrow \infty .
$$

\section{CHIRAL EFFECTIVE FIELD THEORY}

The $\gamma N \rightarrow \Delta$ transition has also been studied within the chiral effective field theory ( $\chi$ EFT) expansions based on chiral Lagrangians with nucleon and $\Delta$-isobar fields. In this framework the expansion for each of the $\gamma^{*} N \Delta$-transition form factors begins with a low-energy constant (LEC), which then receives the chiral loop corrections of the type depicted in Fig. 4.

A first such study was performed by Butler, Savage and Springer [56] in the framework of heavy-baryon $\chi$ PT [57]. At leading order they obtained a "chiral $\log$ " (i.e., $\ln m_{\pi}$ ) enhancement of the $E 2$ transition, which lead to relatively large and positive values of $R_{E M}$. Their result thus showed that the chiral correction to the $E 2$ transition diverges in the chiral limit.

A more comprehensive study was subsequently carried out by Gellas et al. [58] using the so-called "small scale 


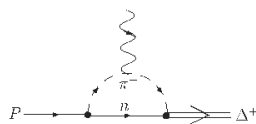

(a)

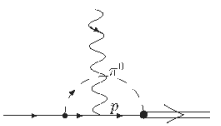

FIGURE 4. The $\pi N$ loop corrections to the $\gamma p \Delta^{+}$vertex as calculated in $\chi$ EFT. Diagram (a) : $\pi^{+} n$ loop where the photon couples to the $\pi^{+}$; diagram (b) : $\pi^{0} p$ loop where the photon couples to the charge of the proton.

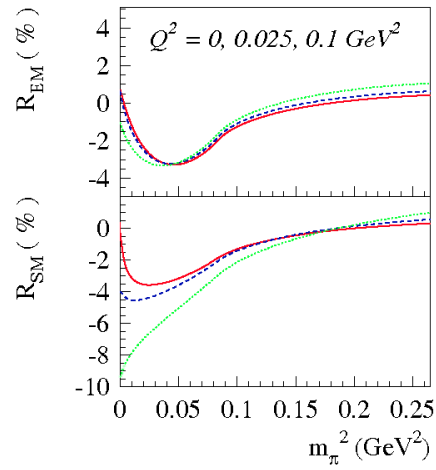

FIGURE 5. The pion mass dependence of $R_{E M}$ and $R_{S M}$ for three fixed values of $Q^{2}: 0$ (solid curves), $0.025 \mathrm{GeV}^{2}$ (dashed curves), and $0.1 \mathrm{GeV}^{2}$ (dotted curves), resulting from the $\chi \mathrm{EFT}$ calculation of Refs. $[62,63]$. The figure shows the absence (presence) of the $\ln m_{\pi}$ divergence for $R_{E M}\left(R_{S M}\right)$ at $Q^{2}=0$.

expansion" (SSE) [59], also called " $\varepsilon$-expansion". In the SSE scheme the two light scales in the problem: the pion mass $\left(m_{\pi}\right)$ and the $\Delta$-resonance excitation energy $\left(\Delta \equiv M_{\Lambda}-M_{N}\right)$ are counted as having the same size. In addition, the heavy-baryon (semi-relativistic) expansion is performed. The calculation of Ref. [58] was performed to order $p^{3}$, which is the next-to-leading order for $M 1$ and leading order for the $E 2$ and $C 2$ transitions. More recently, Gail and Hemmert $[60,61]$ have updated this work and analyzed some of the higher-order contributions. In overall, they show a good agreement with the low $Q^{2}$ data for the $\gamma N \rightarrow \Delta$ transition, as will be illustrated below. However, as a note of caution, one should point out that the $R_{E M}$ and $R_{S M}$ ratios are reasonably well described only upon adding a nominally higher-order $\left(p^{5}\right)$ counter term with an unnaturally large LEC $\left(C_{s} \simeq-17 \mathrm{GeV}^{-2}\right.$ in the notation of Ref. [60]).

In Refs. [62,63], the $\gamma^{*} N \Delta$ form factors have been calculated in a manifestly covariant (no heavy-baryon expansions) $\chi$ EFT expansion, see also [64]. In the chiral limit, it was found that the $C 2$ transition diverges for $Q^{2}=0$, which is also in agreement with the result of Ref. $[60]^{2}$. The coefficient of the chiral log turns out to be small. Only for tiny pion masses one starts to see the dominance of the chiral log in the $C 2$ transition, see Fig. 5 .

The $\chi$ EFT study of the $\gamma N \Delta$ transition in Refs. [62,63] utilizes the " $\delta$-expansion" scheme of Ref. [65]. In this scheme the two light scales $\delta \equiv \Delta / \Lambda_{\chi \mathrm{SB}}$ and $\varepsilon \equiv m_{\pi} / \Lambda_{\chi \mathrm{SB}}$, with $\Lambda_{\chi \mathrm{SB}} \sim 1 \mathrm{GeV}$ the chiral symmetry breaking scale, are treated differently. In contrast, the SSE assumes $\varepsilon \sim \delta$, which leads to an unsatisfactory feature that the $\Delta$-resonance contributions are always estimated to be of the same size as the nucleon contributions (hence, overestimating the $\Delta$

${ }^{2}$ This result of both Refs. [60] and [62, 63] is in disagreement with Butler et al. [56] where a chiral log enhancement was reported for $E 2$ at $Q^{2}=0$ instead of $C 2$. 

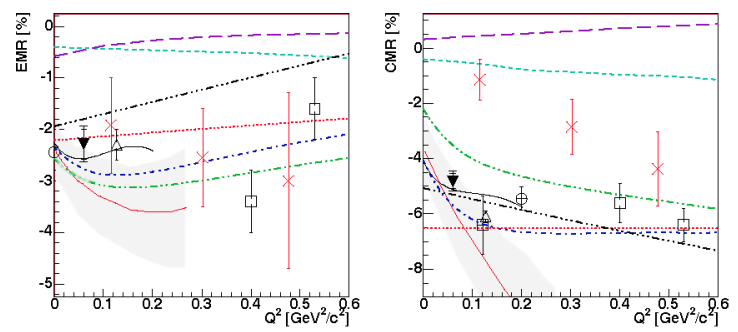

FIGURE 6. The low $Q^{2}$ dependence of the quadrupole ratios $E M R$ (left panel) and $C M R$ (right panel) for the $\gamma^{*} p \rightarrow \Delta$ transition. The data are from MAMI : Refs. $\bigcirc[11], \oplus[66], \boxplus[67], \nabla[68]$; from Bates $\triangle$ [69]; and from CLAS $\square$ [70]. The lattice QCD calculations with linear pion mass extrapolations are shown as $\times[71]$. Two chiral EFT calculations are shown: the $\delta$-expansion result $[62,63]$ (red solid curves with error estimates), and the $\varepsilon$-expansion result [60] (black solid curves). The dynamical model predictions from SL [72] (green dashed-double-dotted curves) and DMT [73] (blue dashed-dotted curves) are shown alongside the MAID2003 [74] (red dotted curves) and SAID [75] (black dashed-triple-dotted curves) models. The hypercentral (long dashed curves) [76] and relativistic (short dashed curves) [28] constituent quark models have been included. Figure from [68].

contribution at lower energies and underestimating some of them at the resonance energies). In the $\delta$-expansion, one counts $\varepsilon=\delta^{2}$, which is the closest integer-power relation between these parameters in the real world.

At the physical pion mass, the $\delta$-expansion provides an energy-dependent power-counting scheme designed to take into account the large variation of the $\Delta$-resonance contributions with energy. As such it allows for an efficient calculation of observables in the resonance region. The relevant LECs can in this fashion be directly extracted from observables. In Refs. $[62,63]$ the three LECs corresponding to the three $\gamma N \Delta$ transitions are all extracted from a nextto-leading (NLO) calculation of pion photo- and electro-production observables. The resulting prediction of the $Q^{2}$ dependence is then shown to be in a qualitative agreement with phenomenological extractions, see Fig. 6 .

Furthermore, it is seen from Fig. 6 that the $R_{S M}$ ratio displays a steep slope at low $Q^{2}$ (Siegert limit), and seems to level off to a negative value around -6 to $-7 \%$ above $Q^{2} \simeq 0.1 \mathrm{GeV}^{2}$. Both the $\chi$ EFT calculations are consistent with the low $Q^{2}$ dependence of the $R_{E M}$ and $R_{S M}$ ratios. The $\delta$-expansion results are quoted with a theoretical uncertainty band as explained in Refs. $[62,63]$. One also sees from Fig. 6 that the constituent quark models largely under-predict the $R_{E M}$ and $R_{S M}$ ratios, as discussed above. The dy namical models of Refs. [72, 73], which include pionic degrees of freedom, are in qualitative agreement with the empirical $Q^{2}$ dependence.

In Fig. 7, a comparison is shown of the $Q^{2}$-dependence arising from the the pion cloud contributions to the $\gamma^{*} N \Delta$ form factors in dynamical models versus $\chi \mathrm{EFT}$. In $\chi \mathrm{EFT}$ the values of the form factors $G_{M}^{*}, G_{E}^{*}$, and $G_{C}^{*}$ at the real photon point are related to three LECs $g_{M}, g_{E}$, and $g_{C}$ appearing in the chiral Lagrangian, see Eq. 2, and determined from a fit to the data. Similarly, in the dynamical models the values at $Q^{2}=0$ are determined from a fit to the same data. However, the renormalization of the pion loop contributions is done in a dramatically different fashion. In $\chi \mathrm{EFT}$ one uses the dimensional regularization and absorbs infinities and other power-counting violating pieces into the LECs. In dynamical models, the loops are regularized by a cutoff, which is then fitted to the data. As the result the pion cloud contributions do not satisfy the chiral power-counting, and are usually larger than the ones obtained from $\chi$ EFT.

Therefore, to compare the dynamical model and $\chi \mathrm{EFT}$ results directly we consider the difference $G_{M}^{*}\left(Q^{2}\right)-G_{M}^{*}(0)$, and analogously for $G_{E}^{*}$ and $G_{C}^{*}$, such that the above renormalization-scheme dependence drops out. In Fig. 7, the results of the dynamical model of Ref. [72] are compared with the $\chi$ EFT results of Ref. [62,63]. For both the dynamical model and $\chi \mathrm{EFT}$, the results with and without the pion-loop effects, are shown which for these difference-quantities appear to be similarly renormalized. From this comparison, one can conclude that the full results, including the pionloop contributions, are very similar in both the dynamical model and the $\chi$ EFT approach, for all the three form factors. The pion cloud contribution to $G_{M}^{*}$ appears to be larger in the dynamical model than in the $\chi \mathrm{EFT}$ calculation. This difference in size is mainly due to the photon coupling to the anomalous magnetic moments of the nucleons in the loops, which are included in the dynamical model, but not in $\chi$ EFT since they are of higher than NLO considered in Refs. [62, 63]. For the Coulomb quadrupole form factor $G_{C}^{*}$, one sees a very similar role of the pion cloud in both approaches, giving rise to a structure around $Q^{2} \simeq 0.03-0.05 \mathrm{GeV}^{2}$. For the electric quadrupole form factor $G_{E}^{*}$, on the other hand, one notices a qualitatively different behavior. Whereas the effect of the pion cloud is very small in the dynamical model calculation, a strong, nearly linear $Q^{2}$ dependence of the pion loops arises in $\chi$ EFT. 


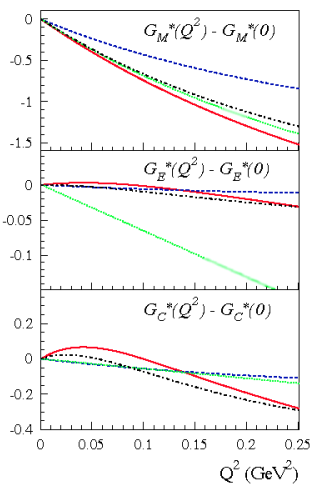

FIGURE 7. Comparison of the pion cloud in the dynamical model and chiral EFT calculations for the low $Q^{2}$ behavior of the Jones Scadron form factors $G_{M}^{*}, G_{E}^{*}$, and $G_{C}^{*}$ (with value at $Q^{2}=0$ subtracted). The dynamical model predictions from SL [72] are shown for both the 'bare' version of the model (blue dashed curves) where the pion loop diagrams are not accounted for, and 'dressed' version of the model (black dashed dotted curves) which include the pion loop diagrams. The chiral EFT calculation of Ref. $[62,63]$ are shown without (green dotted curves) and with (red solid curves) the pion loop diagrams.

\section{LATTICE QCD AND CHIRAL EXTRAPOLATION}

\section{Lattice simulations}

Lattice QCD calculations of nucleon structure quantities have matured considerably in the recent past. They provide an $a b$ initio calculation of quantities such as the $\gamma^{*} N \Delta$ transition form factors from the underlying theory of QCD. The calculation of the $\gamma^{*} N \Delta$ form factors requires the evaluation of three-point functions. The first such calculation by Leinweber, Draper and Woloshyn [77] evaluated these in the so-called fixed current approach, which requires the current to have a fixed direction and to carry a fixed momentum. The initial and final states, on the other hand, can vary without requiring further inversions, which are the time-consuming part of the evaluation of three-point functions. For rather large quark masses, corresponding with pion masses in the range $m_{\pi} \simeq 0.65-0.95 \mathrm{GeV}$, they obtained [77]: $R_{E M}=(-3 \pm 8) \%$. This initial result clearly indicates the need for high statistics to establish a non-zero value for a quantity such as $R_{E M}$.

Alexandrou, de Forcrand and Tsapalis [78] studied the deformation of baryons with spin higher than $1 / 2$ in lattice QCD via three-density correlators, see Ref. [79]. The unquenched lattice QCD calculation indicates a slightly oblate deformation for the $\Delta^{+}$in a spin $+3 / 2$ state, in agreement with the negative value of the $\Delta^{+}$quadrupole moment obtained from the large $N_{c}$ estimate of Eq. (30). It will be very interesting to check if this signal can be consolidated by higher statistics results using lighter quark masses and a larger lattice.

A direct calculation of the $\gamma^{*} N \Delta$ transition form factors on the lattice has also been performed recently. Based on the method of Ref. [77], the Nicosia-MIT group [80, 71] has performed a high-statistics calculations of the $\gamma^{*} N \Delta$ form factors both in quenched and unquenched lattice QCD. As an example, Fig. 8 shows the quenched lattice QCD results of Ref. [71] for the $\gamma^{*} N \Delta$ ratios $R_{E M}$ and $R_{S M}$, together with a linear extrapolation in $m_{\pi}^{2}$. These values are confirmed by unquenched lattice calculations, see [79], in the same pion mass range. However, at present the unquenched calculations are more noisy. For the $R_{E M}$ ratio, the lattice results are accurate enough to show a negative value, which becomes more negative as one approaches the chiral limit. The linearly extrapolated lattice results seem to be in good agreement with the experiment, although the lattice results at larger $Q^{2}$ values show some scatter. The $R_{S M}$ ratio is clearly negative over the whole $Q^{2}$ range. For $Q^{2} \gtrsim 0.5 \mathrm{GeV}^{2}$, the linearly extrapolated lattice results are in agreement with experiment. At lower $Q^{2}$ on the other hand, the linearly extrapolated lattice results fall increasingly short of the data, and cannot explain the large negative value of $R_{S M}$ established in experiment. The present empirical 
results for $R_{S M}$ at low $Q^{2}$ have reached a high level of accuracy and have been cross-checked by several experiments at both Bates and MAMI, which all obtain a rather large negative $R_{S M}$ ratio of around $-6 \%$ at low $Q^{2}$. This puzzle was studied in Refs. $[60,62,63]$ within the framework of $\chi \mathrm{EFT}$, yielding some interesting results as is discussed in the following.
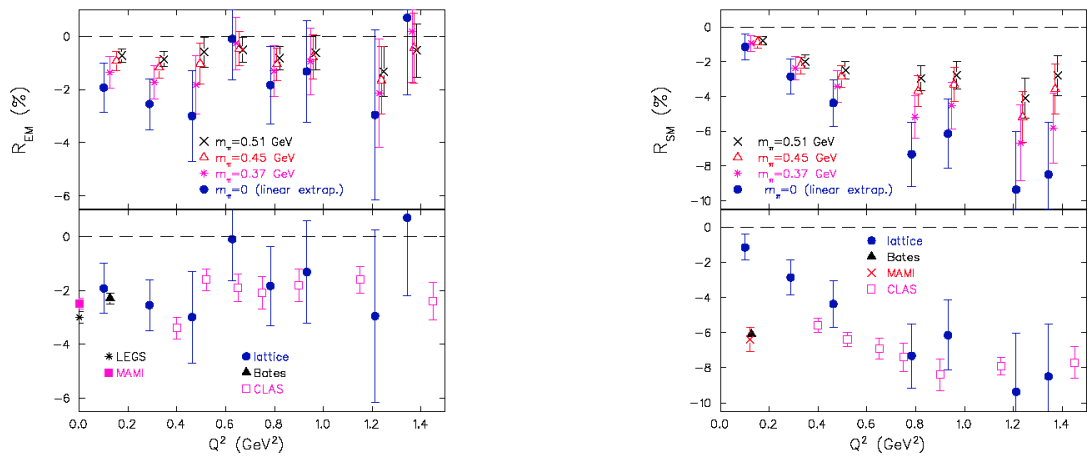

FIGURE 8. Quenched lattice QCD results for the $Q^{2}$ dependence of the $\gamma^{*} N \Delta$ ratios $R_{E M}$ (left panels) and $R_{S M}$ (right panels), for three values of $m_{\pi}$ as indicated on the figure. The (blue) solid circles are the result of a linear extrapolation in $m_{\pi}^{2}$. In the lower panels a comparison with experimental data is shown: real photon points from MAMI [11] and LEGS [13]; finite $Q^{2}$ values are from BATES [81, 69] (solid triangles), MAMI [67] (cross), and JLab/CLAS [70] (open boxes). Figure from Ref. [71].

\section{Chiral extrapolations}

The extrapolation in the quark mass $m_{q}$ is not straightforward, because the non-analytic dependencies, such as $\sqrt{m_{q}}$ and $\ln m_{q}$, become important as one approaches the small physical value of $m_{q}$. Therefore naive extrapolations often fail, while spectacular non-analytic effects are found in a number of different quantities, see $e . g .$, Refs. [82, 83]. 'l'he $\chi$ EFT provides a framework to compute these non-analytic terms. As an example, we first consider the quark mass dependence of the nucleon and $\Delta$ masses within $\chi$ EFT, which have been discussed extensively in the literature, see e.g. Refs. $[84,85,86,87,88,89,90,91]$.

In the $\chi E F T$ including $\Delta$ degrees of freedom, the pion mass dependence of $N$ and $\Delta$ masses near the chiral limit, i.e. for $m_{\pi}<\Delta \equiv M_{\Delta}-M_{N}$, goes as [84]:

$$
\begin{aligned}
& M_{N}=M_{N}^{(0)}-4 c_{1 N} m_{\pi}^{2}-\frac{3}{32 \pi f_{\pi}^{2}} g_{A}^{2} m_{\pi}^{3}+\frac{2}{\left(8 \pi f_{\pi}\right)^{2}} h_{A}^{2} \frac{m_{\pi}^{4}}{\Delta} \ln m_{\pi}+O\left(m_{\pi}^{4}\right), \\
& M_{\Delta}=M_{\Delta}^{(0)}-4 c_{1 \Delta} m_{\pi}^{2}-\frac{3}{32 \pi f_{\pi}^{2}} \frac{25}{81} H_{A}^{2} m_{\pi}^{3}-\frac{1}{2\left(8 \pi f_{\pi}\right)^{2}} h_{A}^{2} \frac{m_{\pi}^{4}}{\Delta} \ln m_{\pi}+O\left(m_{\pi}^{4}\right) .
\end{aligned}
$$

The non-analytic terms (proportional to $m_{\pi}^{3}, m_{\pi}^{4} / \Delta \ln m_{\pi}, \ldots$ ) on the $r h s$ of Eqs. (33,34) are predictions of $\chi$ EFT obtained from the one-loop pion diagrams. These loop expressions depend on the coupling constants appearing in the lowest order chiral Lagrangian : $g_{A}=1.267$ is the axial coupling of the nucleon, $h_{A} \simeq 2.85$ is the $\pi N \Delta$ coupling constant, and $H_{A}$ is the axial $\pi \Delta \Delta$ coupling constant, which is related with $g_{A}$ through the $S U(6)$ relation, which coincides with the large $N_{c}$ relation: $H_{A}=(9 / 5) g_{A} \simeq 2.28$. The analytic terms on the $r h s$ of Eqs. $(33,34)$ are low-energy constants which have to be determined from experiment or from a fit to lattice QCD results. In particular $M_{N}^{0}\left(M_{\Delta}^{0}\right)$ is the $N(\Delta)$ mass in the chiral limit, and the term proportional to $c_{1 N}\left(c_{1 \Delta}\right)$ is the quark mass contribution to the $N(\Delta)$ mass. For the nucleon, it is obtained from the experimental information on the pion-nucleon $\sigma$-term.

The above formulas of Eqs. $(33,34)$ can be fitted to full lattice QCD results for the $N$ and $\Delta$ masses. Care has to be taken however when fitting to quenched lattice QCD results, where sea quark loop effects are neglected. The quenched approximation also modifies the leading chiral expansion of baryon masses in the corresponding effective field theory, see Ref. [92]. For instance, in the quenched approximation, the pion mass dependence of $N$ and $\Delta$ masses contain a 


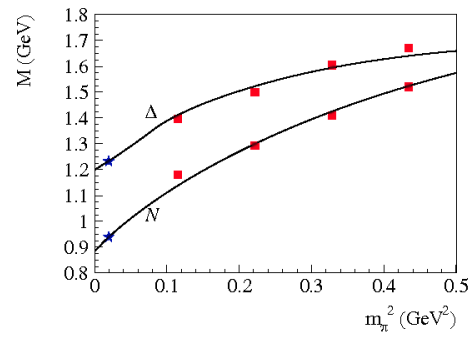

FIGURE 9. Pion-mass dependence of the $N$ and $\Delta$ masses. The curves are two-parameter expressions for $M_{N}$ and $M_{\Delta}$ according to the manifestly covariant $\chi$ EFT framework of [91]. The red squares are lattice results from the MILC Collaboration [93] in full QCD. The stars represent the physical mass values.

term linear in $m_{\pi}$, whereas such a term is absent in the full QCD expansions of Eqs. $(33,34)$.

To account for higher order terms in the chiral expansion, the $m_{\pi}$ dependence of $M_{N}$ and $M_{\Delta}$ has been studied in Ref. [91] in a manifestly covariant $\chi$ EFT framework consistent with analyticity. The resulting relativistic loop corrections obey the chiral power-counting, after renormalizations of the available counter-terms are done. The relativistic expressions also contain the nominally higher-order terms, which are necessary to satisfy the analyticity constraint. In such approach, the analytic terms in the quark mass arising from the one-loop pion diagrams are partially resummed. It was found [91] that the convergence of the chiral expansion for $M_{N}$ and $M_{\Delta}$ may be improved without introducing additional parameters. In Fig. 9, the results for the $m_{\pi}$ dependence of $N$ and $\Delta$-resonance masses in this approach are compared with full lattice QCD results. For both $N$ and $\Delta$, the chiral limit mass values $M_{N}^{(0)}\left(M_{\Delta}^{(0)}\right)$ and the parameters $c_{1 N}\left(c_{1 \Delta}\right)$ are fitted. As is seen from the figure, such a two-parameter form for $M_{N}$ and $M_{\Delta}$, a good description of lattice results is obtained up to $m_{\pi}^{2} \simeq 0.5 \mathrm{GeV}^{2}$. It should be stressed however that such large values of $m_{\pi}$ are well outside the regime where a perturbative chiral expansion can be expected to hold.

Analogously, the $m_{\pi}$ dependence of $G_{M}^{*}$, and of the ratios $R_{E M}$ and $R_{S M}$, have been calculated with chiral EFT, see Refs. [61, 64]. As discussed above, the recent state-of-the-art latlice calculations of $R_{E M}$ and $R_{S M}$ [71] use a linear, in the quark mass $\left(m_{q} \propto m_{\pi}^{2}\right)$, extrapolation to the physical point, thus assuming that the non-analytic $m_{q^{-}}$dependencies are negligible. The thus obtained value for $R_{S M}$ at the physical $m_{\pi}$ value displays a large discrepancy with the experimental result, as seen in Fig. 8. The relativistic $\chi$ EFT calculation $[62,63]$, on the other hand, shows that the non-analytic dependencies are not negligible. While at larger values of $m_{\pi}$, where the $\Delta$ is stable, the ratios display a smooth $m_{\pi}$ dependence, at $m_{\pi}=\Delta$ there is an inflection point, and for $m_{\pi} \leq \Delta$ the non-analytic effects are crucial, as was also observed for the $\Delta$-resonance magnetic moment $[94,95]$.

To shed further light on the predicted strong $m_{\pi}$ dependence of the $\gamma^{*} N \Delta$ form factors, new lattice calculations, within full QCD, are underway [79]. The full QCD results obtained so far are more noisy but in agreement with those obtained in the quenched theory. It will be interesting to test the predicted strong non-analytic effects in the $R_{E M}$ and $R_{S M}$ ratios once high statistics full lattice QCD results for pion masses smaller than $0.3 \mathrm{GeV}$ become available.

\section{GENERALIZED PARTON DISTRIBUTIONS (GPDS)}

\section{Definition of $N \rightarrow \Delta$ GPDs and form factor sum rules}

So far, the $N \rightarrow \Delta$ transition was discussed as revealed with the help of the electromagnetic probe. By measuring the response of the hadron to a virtual photon, one measures the matrix element of a well-defined quark-gluon operator (in this case the vector operator $\bar{q} \gamma^{\mu} q$ ) over the hadronic state. This matrix element can be parametrized in terms of the $\gamma^{*} N \Delta$ transition form factors, revealing the quark-gluon structure of the hadron. We are however not limited in nature to probes such as photons (or $W, Z$ bosons for the axial transition). The phenomenon of asymptotic freedom of QCD, meaning that at short distances the interactions between quarks and gluons become weak, provides 


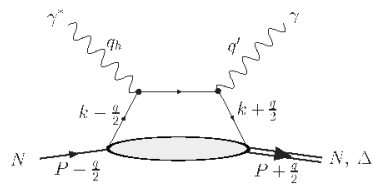

FIGURE 10. The "handbag" diagram for the $N \rightarrow N$ and $N \rightarrow \triangle$ DVCS processes. Provided the virtuality of the initial photon (with momentum $q_{h}$ ) is sufficiently large, the QCD factorization theorem allows to express the total amplitude as the convolution of a Compton process at the quark level and a non-perturbative amplitude parametrized in terms of generalized parton distributions (lower blob). The diagram with the photon lines crossed is also understood.

us with more sophisticated QCD operators to explore the structure of hadrons. Such operators can be accessed by selecting a small size configuration of quarks and gluons, provided by a hard reaction, such as deep inelastic scattering (DIS), or hard exclusive reactions such as deeply virtual Compton scattering (DVCS), $\gamma^{*}\left(q_{h}\right)+N(p) \rightarrow \gamma\left(q^{\prime}\right)+B\left(p^{\prime}\right)$, where the virtual photon momentum $q_{h}$ is the hard scale, and where the final state $B$ stands for either the nucleon $N$ or the $\Delta$ state. The common important feature of such hard reactions is the possibility to separate clearly the perturbative and nonperturbative stages of the interactions, this is the so-called factorization property. It is illustrated in Fig. 10 for the case of the DVCS process with a $\Delta$ in the final state. The non-perturbative stage of such hard exclusive electroproduction processes is described by universal objects, so-called generalized parton distributions (GPDs) $[96,97,98]$, see Refs. [99, 100, 101, 102] for reviews and references.

The GPDs depend in general on three variables: $x, \xi$, and $Q^{2}$. The light-cone momentum fraction $x$ is defined by $k^{+}=x P^{+}$, where $k$ is the quark loop momentum and $P$ is the average nucleon momentum $P=\left(p+p^{\prime}\right) / 2$, where $p\left(p^{\prime}\right)$ are the initial (final) baryon four-momenta, see Fig. 10. The skewedness variable $\xi$ is defined by $q^{+}=-2 \xi P^{+}$, where $q=p^{\prime}-p$ is the overall momentum transfer in the process, and where $2 \xi \rightarrow x_{B} /\left(1-x_{B} / 2\right)$ in the Bjorken limit; $x_{B}=Q_{h}^{2} /\left(2_{p} \cdot q_{h}\right)$ is the usual Bjorken scaling variable, with $Q_{h}^{2}=-q_{h}^{2}>0$ the virtuality of the hard photon. Finally, the third variable is the total momentum transfer squared: $Q^{2}=-q^{2}$.

In a frame where the virtual photon momentum $q_{h}$ and the average nucleon momentum $P$ are along the $z$-axis and opposite to each other, one can parameterize the non-perturbative piece of the $N \rightarrow \Delta$ DVCS amplitude as [100,105]:

$$
\begin{aligned}
& \left.\frac{1}{2 \pi} \int d y^{-} e^{i x P^{+} y^{-}}\left\langle\Delta\left|\bar{\psi}\left(-\frac{y}{2}\right) \gamma \cdot n \tau_{3} \psi\left(\frac{y}{2}\right)\right| N\right\rangle\right|_{y^{+}=\vec{y}_{\perp}=0} \\
& =\sqrt{\frac{2}{3}} u^{\alpha}\left(p^{\prime}\right)\left\{H_{M}\left(-\mathscr{K}_{\alpha \mu}^{M}\right)+H_{E}\left(-\mathscr{K}_{\alpha \mu}^{E}\right)+H_{C}\left(-\mathscr{K}_{\alpha \mu}^{C}\right)+H_{4}\left(g_{\alpha \mu} \gamma_{5}\right)\right\} n^{\mu} u(p),
\end{aligned}
$$

where $\psi$ is the quark field of flavor $q, u$ the nucleon spinor, $u^{\alpha}\left(p^{\prime}\right)$ is the Rarita-Schwinger spinor for the $\Delta$-field, $n^{\mu}$ is a light-cone vector along the negative $z$-direction, $\tau_{3} / 2$ is the third isospin generator for quarks, and $\sqrt{2 / 3}$ is the isospin factor for the $p \rightarrow \Delta^{+}$transition. Furthermore, in Eq. (35), the covariants $\mathscr{K}_{\alpha \mu}^{M, E, C}$ are the magnetic dipole, electric quadrupole, and Coulomb quadrupole Jones-Scadron covariants [8]:

$$
\begin{aligned}
& \mathscr{K}_{\alpha \mu \mu}^{M}=-i \frac{3\left(M_{\Delta}+M_{N}\right)}{2 M_{N} Q_{+}^{2}} \varepsilon_{\alpha \mu \lambda \sigma} P^{\lambda} q^{\sigma}, \\
& \mathscr{K}_{\alpha \mu}^{E}=-\mathscr{K}_{\alpha \mu}^{M}-\frac{6\left(M_{\Delta}+M_{N}\right)}{M_{N} Q_{+}^{2} Q_{-}^{2}} \varepsilon_{\alpha \sigma \lambda \rho} P^{\lambda} q^{\rho} \varepsilon_{\mu \kappa \delta}^{\sigma} P^{\kappa} q^{\delta} \gamma_{5}, \\
& \mathscr{K}_{\alpha \mu}^{C}=-i \frac{3\left(M_{\Delta}+M_{N}\right)}{M_{N} Q_{+}^{2} Q_{-}^{2}} q_{\alpha}\left(q^{2} P_{\mu}-q \cdot P q_{\mu}\right) \gamma_{5} .
\end{aligned}
$$

The thus introduced GPDs $H_{M}, H_{E}$, and $H_{C}$, functions of $x, \xi$, and $Q^{2}$, correspond with the three $N \rightarrow \Delta$ Jones-Scadron form factors and relate to them via the sum rules:

$$
\int_{-1}^{1} d x H_{M, E, C}\left(x, \xi, Q^{2}\right)=2 G_{M, E, C}^{*}\left(Q^{2}\right)
$$

The fourth $N \rightarrow \Delta$ vector GPD $H_{4}$ in Eq. (35) has a vanishing first moment.

The above sum rules allow one to make a prediction for the $N \rightarrow \Delta$ form factors provided one has a model for the 


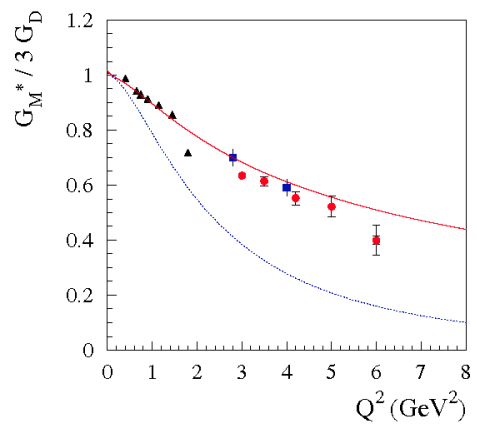

FIGURE 11. The $N \rightarrow \Delta$ magnetic transition (Jones-Scadron) form factor $G_{M}^{*}$, relative to the dipole form (multiplied by a factor 3). The curves show two parametrizations of the $N \rightarrow \Delta$ magnetic GPD $H_{M}$ of Ref. [108] : a one-parameter Regge GPD parameterization (blue dotted curve) and a 3-parameter modified Regge GPD parametrization (red solid curve). The data points are from JLab Hall C [111] (blue squares), and JLab CLAS : Refs. [70] (black triangles) and [112] (red circles).

$N \rightarrow \Delta$ GPDs. Conversely, the existing precise experimental information on the $N \rightarrow \Delta$ vector form factors provides a strong constraint on the $N \rightarrow \Delta$ GPDs. The GPDs are however much richer observables than form factors and provide us with quark distribution information in the $\Delta$ resonance. They can be accessed by the $N \rightarrow \Delta$ DVCS process as discussed in Ref. [103]. First experiments which are sensitive to the $N \rightarrow \Delta$ GPDs have recently been reported [104].

\section{Model for the $N \rightarrow \Delta$ GPDs and transverse structure of hadrons}

For the magnetic dipole $N \rightarrow \Delta$ transition, it was shown that, in the large- $N_{c}$ limit, the relevant $N \rightarrow \Delta$ GPD $H_{M}$ can be expressed in terms of the nucleon isovector GPD $E^{\mathfrak{u}}-E^{d}$ as [105] :

$$
H_{M}=2 \frac{G_{M}^{*}(0)}{\kappa_{V}}\left\{E^{\mathfrak{u}}-E^{d}\right\}
$$

where $\kappa_{V}=\kappa_{p}-\kappa_{n}=3.70$ is the nucleon isovector anomalous magnetic moment. The large- $N_{c}$ limit value $G_{M}^{*}(0)=$ $\kappa_{V} / \sqrt{2}$ is about $20 \%$ smaller than the phenomenological value $G_{M}^{*}(0) \approx 3.02$ of Eq. (15), used in the following.

Using the large $N_{c}$ estimate of Eq. (38), the sum rule Eq. (37) for $G_{M}^{*}$ can be written as:

$$
G_{M}^{*}\left(Q^{2}\right)=\frac{G_{M}^{*}(0)}{\kappa_{V}} \int_{-1}^{+1} d x\left\{E^{u}\left(x, \xi, Q^{2}\right)-E^{d}\left(x, \xi, Q^{2}\right)\right\}=\frac{G_{M}^{*}(0)}{\kappa_{V}}\left\{F_{2}^{p}\left(Q^{2}\right)-F_{2}^{n}\left(Q^{2}\right)\right\},
$$

where $F_{2}^{p}-F_{2}^{n}$ is the isovector combination of the proton (p) - neutron (n) Pauli form factors. Because the sum rule of Eq. (39) is independent of $\xi$, we only need to constrain the nucleon GPD $E^{q}$ for $\xi=0$ in order to evaluate $G_{M}^{*}$. The sum rule (39) was used in Ref. [106], using a model in which a Gaussian ansatz for nucleon GPDs is modified at large $Q^{2}$ by terms having a power-law behavior. Refs. [107, 108, 109] used parametrizations which are motivated from the expected Regge behavior of the GPDs at small $x$ and $Q^{2}$. In Ref. [108], a 3-parameter modified Regge parametrization for the nucleon GPDs was found to provide a good quantitative description for all four nucleon elastic form factors over the whole $Q^{2}$ range. Using the sum rule prediction based on the large $N_{c}$ estimate of Eq. (39), the $N \rightarrow \Delta$ magnetic FF $G_{M}^{*}$ follows as a prediction. It is seen from Fig. 11 that the large $N_{c}$ relation reproduces well the experimentally observed, faster than dipole, fall-off of $G_{M}^{*}$. This is also in agreement with the corresponding fall-off of the nucleon isovector Pauli form factor, confirming the finding of Ref. [106] using a Gaussian model for the GPDs. 
In Ref. [110], such large $N_{c}$ relations have been extended to the $N \rightarrow \Delta$ GPDs for the electric and Coulomb quadrupole transitions. Starting from a large $N_{c}$ relation, Eq.(31), between the $N \rightarrow \Delta$ quadrupole moment $Q_{p \rightarrow \Delta^{+}}$ and the neutron charge radius $r_{n}^{2}$, one can express it equivalently as a relation for $G_{E}^{*}(0)$ (to leading order in the $1 / N_{c}$-expansion) as:

$$
G_{E}^{*}(0)=-\frac{M_{\Delta}^{2}-M_{N}^{2}}{12 \sqrt{2}} r_{n}^{2}
$$

For small values of $Q^{2}$, the neutron electric form factor is expressed as $G_{E}^{n}\left(Q^{2}\right) \approx-r_{n}^{2} Q^{2} / 6$. Therefore, an extension of the large- $N_{c}$ relation (40) to finite $Q^{2}$ is given by:

$$
G_{E}^{*}\left(Q^{2}\right) \simeq \frac{1}{\sqrt{2}} \frac{M_{\Delta}^{2}-M_{N}^{2}}{2 Q^{2}} G_{E}^{n}\left(Q^{2}\right) .
$$

An analogous prediction for the Coulomb quadrupole $N \rightarrow \Delta \mathrm{GPD}, H_{C}$, can be made by using the large $N_{c}$ relation of Eq. (32). Extending this relation to finite $Q^{2}$ yields

$$
G_{C}^{*}\left(Q^{2}\right) \simeq \frac{4 M_{\Delta}^{2}}{M_{\Delta}^{2}-M_{N}^{2}} G_{E}^{*}\left(Q^{2}\right)
$$

which can be turned into a relation between the $R_{E M}$ and $R_{S M}$ ratios, using Eq. (11), as :

$$
R_{S M}\left(Q^{2}\right) \approx \frac{Q_{+} Q_{-}}{M_{\Delta}^{2}-M_{N}^{2}} R_{E M}\left(Q^{2}\right)
$$

At $Q^{2}=0$, one recovers the relation $R_{S M}=R_{E M}$, Eq. (32).

The form factors $G_{E}^{*}$ and $G_{C}^{*}$ are obtained from the first moment of the $N \rightarrow \Delta$ GPDs $H_{E}$ and $H_{C}$ through the sum rules of Eq. (37). We can therefore use Eqs. (41) and (42) to obtain relations between the $N \rightarrow \Delta$ GPDs $H_{E}$ and $H_{C}$ and the neutron electric GPD combination as :

$$
H_{E}=\frac{1}{\sqrt{2}} \frac{M_{\Delta}^{2}-M_{N}^{2}}{Q^{2}}\left\{H^{(n)}-\frac{Q^{2}}{4 M_{N}^{2}} E^{(n)}\right\}, \quad H_{C}=\frac{4 M_{\Lambda}^{2}}{M_{\Delta}^{2}-M_{N}^{2}} H_{E},
$$

where the (neutron) GPDs $H^{(n)}$ and $E^{(n)}$ are defined in terms of the $u$ - and $d$-quark flavor GPDs as: $H^{(n)} \equiv-1 / 3 H^{u}+$ $2 / 3 H^{d}$, and $E^{(n)} \equiv-1 / 3 E^{u}+2 / 3 E^{d}$.

The predictions which follows from the large- $N_{c}$ motivated expressions, Eqs. $(41,43)$, are tested in Fig. 12 by comparing the $Q^{2}$ dependence of the neutron electric FF $G_{E}^{n}$ and the $N \rightarrow \Delta R_{E M}$ and $R_{S M}$ ratios. Although the above relations are derived assuming small $Q^{2}$, we explore their empirical validity at moderate $Q^{2}$ as well. The neutron electric form factor $G_{E}^{n}$ is computed using the modified Regge parametrization of [108], which is seen to give a fairly good description of the available double polarization data. The $R_{E M}$ and $R_{S M}$ ratios are then computed using the above large- $N_{C}$ relations. By using the three parameter Regge form for the nucleon GPDs, one thus obtains a parameterfree predictions for $R_{E M}$ and $R_{S M}$. One sees that this yields a $R_{E M}$ ratio which has both the right size and displays a relatively flat $Q^{2}$ behavior, up to $Q^{2}$ of about $2 \mathrm{GeV}^{2}$. The prediction of the $Q^{2}$ dependence for both ratios is in a surprisingly (for a large- $N_{c}$ estimate) good agreement with the experimental data.

The interplay between the $x$ and $Q^{2}$-dependence of the GPDs contains new nucleon structure information beyond the information encoded in forward parton distributions depending only on $x$, or form factors depending only on $Q^{2}$. It has been shown that by a Fourier transform of the $Q^{2}$-dependence of GPDs, it is conceivable to access the distributions of parton in the transverse plane, see Ref. [115], and to provide a 3-dimensional picture of the nucleon [116].

For $\xi=0$, one can define the impact parameter versions of GPDs which are obtained through a Fourier integral in transverse momentum $q_{\perp}$. For the GPD $E^{q}$, this reads as:

$$
E^{q}\left(x, \mathbf{b}_{\perp}\right) \equiv \int \frac{d^{2} \mathbf{q} \perp}{(2 \pi)^{2}} e^{i \mathbf{b}_{\perp} \cdot \mathbf{q}_{\perp}} E^{q}\left(x, 0,-\mathbf{q}_{\perp}^{\mathbf{2}}\right),
$$

and analogous definitions for the other GPDs. These impact parameter GPDs have the physical meaning of measuring the probability to find a quark which carries longitudinal momentum fraction $x$ at a transverse position $\mathbf{b}_{\perp}$ (relative to 


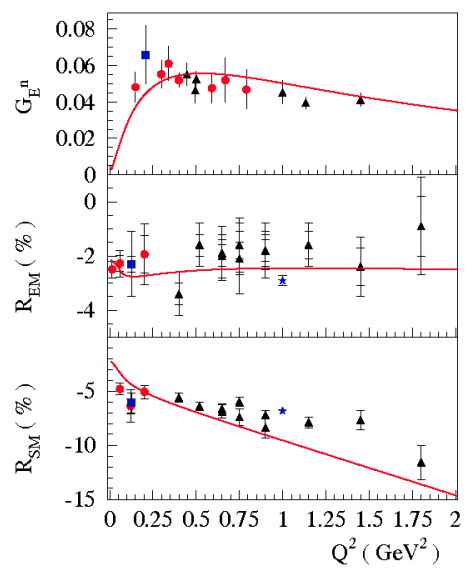

FIGURE 12. (Color online) Neutron electric form factor $G_{E}^{n}$ (upper panel) in comparison with the $N \rightarrow \Delta R_{E M}$ (middle panel) and $R_{S M}$ (lower panel) ratios. The curves result from the modified Regge GPD parametrization [108], where in computing $R_{E M}$ and $R_{S M}$, the large- $N_{c}$ relations $(39,41,42)$ are used. Data points for $G_{F}^{n}$ are from MAMI (red circles), NIKHEF (blue square), and JLab (black triangles), see [108] for references. Data points for $R_{E M}$ and $R_{S M}$ are from BATES [69] (blue squares), MAMI [11, 68, 113] (red circles), JLab/CLAS [70] (black triangles), and JLab/HallA [114] (blue stars).

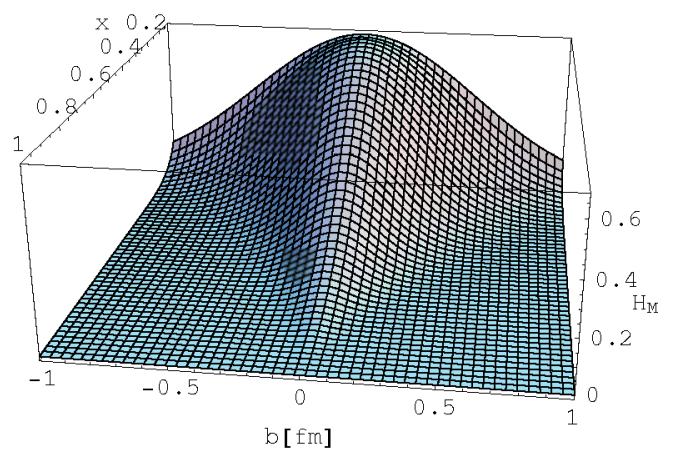

FIGURE 13. The magnetic dipole $N \rightarrow \Delta$ GPD $H_{M}$ as function of the quark momentum fraction $x$ and the quark position $b$ in the transverse plane (perpendicular to the average direction of the fast moving baryons), where $b$ stands for either $\left(\mathbf{b}_{\perp}\right)_{x}$ or $\left(\mathbf{b}_{\perp}\right)_{y}$. The calculation is based on the large $N_{c}$ relation (46), using the modified Regge GPD parametrization for the nucleon GPDs $E$.

the transverse center-of-momentum) in a nucleon, see Refs. [115, 117].

Using the large $N_{c}$ relation Eq. (38) we can then express the impact parameter version of the magnetic $N \rightarrow \Delta$ GPD $H_{M}$ as:

$$
H_{M}\left(x, \mathbf{b}_{\perp}\right)=2 \frac{G_{M}^{*}(0)}{\kappa_{V}}\left\{E^{u}\left(x, \mathbf{b}_{\perp}\right)-E^{d}\left(x, \mathbf{b}_{\perp}\right)\right\} .
$$




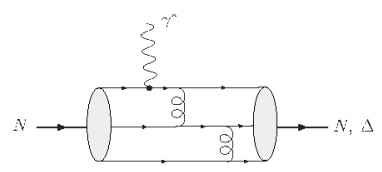

FIGURE 14. Perturbative QCD picture for the nucleon elastic and $N \rightarrow \Delta$ transition electromagnetic form factors. The highly virtual photon resolves the leading three-quark Fock states of $N$ and $\Delta$, described by a distribution amplitude. The large momentum is transferred between the quarks through two gluon exchanges (only one of several possible lowest-order diagrams is shown).

Fig. 13 displays the impact parameter magnetic GPD $H_{M}\left(x, \mathbf{b}_{\perp}\right)$ using the modified Regge GPD parametrization discussed above. It is clearly seen from this image that for large values of $x$, the quark distributions are concentrated at small values of $\mathbf{b}_{\perp}$, reflecting the distribution of valence quarks in the core of the $N$ and $\Delta$. On the other hand, at small values of $x$, the distribution in transverse position extends much further out.

\section{Perturbative QCD (pQCD)}

The electro-excitation of the $\Delta$ provides a famous test for perturbative QCD, where scaling and selection rules for dominant helicity amplitudes were derived and are expected to be valid at sufficiently high momentum transfers $Q^{2}$ [118]. A photon of sufficient high virtuality will see a nucleon (or $\Delta$ ) consisting of three massless quarks moving collinear with the nucleon. When measuring an elastic nucleon form factor or a $N \rightarrow \Delta$ transition form factor, the final state consists again of three massless collinear quarks. In order for this (unlikely process) to happen, the large momentum of the virtual photon has to be transferred among the three quarks through two hard gluon exchanges as illustrated in Fig. 14. This hard scattering mechanism is generated by valence quark configurations with small transverse size and finite light cone momentum fractions of the total hadron momentum carried by each valence quark. The hard amplitude can be written in a factorized form $[119,120,121,118]$, as a product of a perturbatively calculable hard scattering amplitude and two distribution amplitudes describing how the large longitudinal momentum of the initial and final hadrons is shared between their constituents. Because each gluon in such hard scattering process carries a virtuality proportional to $Q^{2}$, this leads to the $\mathrm{pQCD}$ prediction that the helicity conserving nucleon Dirac form factor $F_{1}$ should fall as $1 / Q^{4}$ (modulo $\ln Q^{2}$ factors) at sufficiently high $Q^{2}$. Processes such as in Fig. 14, where the interactions among the quarks proceed via gluon or photon exchange, both of which are vector interactions, conserve the quark helicity in the limit when the quark masses or off-shell effects can be neglected. In contrast to the helicity conserving form factor $F_{1}$, the nucleon Pauli form factor $F_{2}$ involves a helicity flip between the initial and final nucleons. Hence it requires one helicity flip at the quark level, which is suppressed at large $Q^{2}$. Therefore, for collinear quarks, i.e. moving in a light-cone wave function state with orbital angular momentum projection $l_{2}=0$ (along the direction of the fast moving hadron), the asymptotic prediction for $F_{2}$ leads to a $1 / Q^{6}$ fall-off at high $Q^{2}$.

Carlson derived analogous relations for the $\gamma^{*} N \Delta$ transition form factors [122]. For the magnetic $\gamma^{*} N \Delta$ form factor $G_{M}^{*}$, one obtains a $1 / Q^{4}$ behavior for $Q^{2} \rightarrow \infty$. For the $E 2$ and $C 2$ transitions, pQCD predicts : $G_{E}^{*} \rightarrow-G_{M}^{*}$ in the limit $Q^{2} \rightarrow \infty$, which yields $R_{E M} \rightarrow+1$ for $Q^{2} \rightarrow \infty$. For $R_{S M}$, pQCD predicts $: R_{S M} \rightarrow$ constant for $Q^{2} \rightarrow \infty$.

One can test how well the above baryon form factor pQCD scaling predictions are satisfied at the available momentum transfers, see Fig. 15. One firstly sees from Fig. 15 that the proton Dirac form factor, which has been measured up to about $30 \mathrm{GeV}^{2}$, displays an approximate $1 / Q^{4}$ scaling above $10 \mathrm{GeV}^{2}$. For the proton ratio $F_{2}^{p} / F_{1}^{p}$, the data up to $5.6 \mathrm{GeV}^{2}$ show no sign of a $1 / Q^{2}$ behavior as predicted by pQCD. Instead, the data show that the ratio $F_{2}^{p} / F_{1}^{p}$ falls less fast than $1 / Q^{2}$ with increasing $Q^{2}$. In Ref. [128], the assumption of quarks moving collinearly, underlying the pQCD prediction, has been investigated. It has been shown in Ref. [128] that by including components in the nucleon light-cone wave functions with quark orbital angular momentum projection $l_{z}=1$, one obtains the behavior $F_{2} / F_{1} \rightarrow \ln ^{2}\left(Q^{2} / \Lambda^{2}\right) / Q^{2}$ at large $Q^{2}$, with $\Lambda$ a non-perturbative mass scale. Choosing $\Lambda$ in the range $0.2-0.4 \mathrm{GeV}$, Ref. [128] found that the data for $F_{2}^{p} / F_{1}^{p}$ support such double-logarithmic enhancement. A same analy sis, including states of orbital angular momentum projection $l_{z}=1$ in the $N$ or $\Delta$ light-cone wave functions, which find their phy sical origin in the transverse momentum of the quarks in the $N$ and $\Delta$, was also performed for the $\gamma^{*} N \Delta$ Coulomb transition form factor $G_{C}^{*}$ [129]. It was shown in Ref. [129] that due to the orbital motion of the partons, $R_{S M}$ acquires a doublelogarithmic correction $\ln ^{2}\left(Q^{2} / \Lambda^{2}\right)$ at large $Q^{2}$ compared with the standard scaling analy sis, according to which $R_{S M} \rightarrow$ 


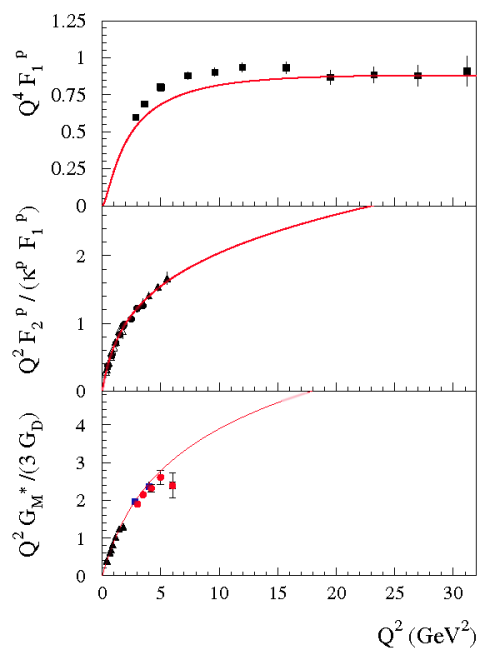

FIGURE 15. Test of baryon form factor scaling predictions. Top panel : proton Dirac form factor multiplied by $Q^{4}$; middle panel : ratio of Pauli to Dirac proton form factors multiplied by $Q^{2}$; lower panel : ratio of the $N \rightarrow \Delta$ magnetic transition (Jones-Scadron) form factor $G_{M}^{*}$ multiplied by $Q^{2}$ to the dipole form factor $G_{D}$. The data for $F_{1}^{p}$ are from [123] (solid squares). Data for the ratio $F_{2}^{p} / F_{1}^{p}$ are from $[124,125]$ (solid circles), [126] (open triangles), and [127] (solid triangles). Data for $G_{M}^{*}$ as in Fig. 11. The red curve is the calculation based on the three parameter modified Regge GPD parametrization of Ref. [108].

constant for $Q^{2} \rightarrow \infty$. The arguments of Refs. [128,129] still rely on $\mathrm{pQCD}$ and it remains to be seen by forthcoming data at higher $Q^{2}$ if this prediction already sets in in the few $\mathrm{GeV}^{2}$ region.

The test of the scaling behavior of the magnetic $\gamma^{*} N \Delta$ form factor $G_{M}^{*}$ is also shown on Fig. 15. As pQCD predicts a $1 / Q^{4}$ asymptotic scaling behavior, the ratio $G_{M}^{*} / G_{D}$ (with $G_{D}$ the dipole form factor) should approach a constant. The data for $G_{M}^{*}$ up to about $6 \mathrm{GeV}^{2}$ again do not support this scaling behavior. One sees instead from Fig. 15 that the data for $G_{M}^{*}$ seem to support a similar $Q^{2}$ behavior as for $F_{2}$. The pQCD prediction for the $R_{E M}$ ratio which should approach $+100 \%$ at high $Q^{2}$ also fails dramatically. The $R_{E M}$ ratio is measured to be minus a few percent out to $Q^{2} \simeq 6 \mathrm{GeV}^{2}$ [112], with no clear indication of a zero crossing. Also the $R_{S M}$ ratio which has also been measured up to $Q^{2} \simeq 6 \mathrm{GeV}^{2}$ [112], does not seem to settle to a constant at large $Q^{2}$.

Although at high enough $Q^{2}$, the pQCD scaling predictions should set in, the available data for the nucleon and $N \rightarrow \Delta$ electromagnetic form factors clearly show that one is still far away from this regime. An explanation has been provided in Refs. [130,131], where it has been argued that the above described hard scattering mechanism is suppressed at accessible momentum transfers relative to the Feynman mechanism [132], also called soft mechanism. The soft mechanism involves only one active quark, and the form factor is obtained as an overlap of initial and final hadron wave functions. The hard scattering mechanism on the other hand, involving three active quarks, requires the exchange of two gluons each of which brings in a suppression factor $\alpha_{s} / \pi \sim 0.1$. One therefore expects the hard scattering mechanism for $F_{1}^{p}$ or $G_{M}^{*}$ to be numerically suppressed by a factor $1 / 100$ compared to the soft term. Even though the soft mechanism is suppressed asymptotically by a power of $1 / Q^{2}$ relative to the hard scattering mechanism, it may well dominate at accessible values of $Q^{2}$.

The above discussed GPDs, represented by the lower blob in Fig. 10, are non-perturbative objects which include higher Fock components in the $N$ and $\Delta$ wave functions. One can use a GPD parametrization to provide an estimate of the soft contributions, and expects this non-perturbative approach to be relevant in the low and intermediate $Q^{2}$ region for the form factors. This is shown in Fig. 15 (solid curves) from which one sees that the GPD parameterization is able to explain at the same time an approximate $1 / Q^{4}$ behavior for $F_{1}^{p}$ and a behavior for $F_{2}^{p} / F_{1}^{p}$ which falls less steep 
than $1 / Q^{2}$. For $G_{M}^{*}$, one sees from Fig. 15 that the GPD sum rule evaluation based on the large $N_{c}$ relation of Eq. (39) is supported by the available data up to about $6 \mathrm{GeV}^{2}$. Forthcoming experiments at the Jefferson Lab $12 \mathrm{GeV}$ facility will extend the data for $F_{2}^{p} / F_{1}^{p}$ and $G_{M}^{*}$ to $Q^{2}$ values around $15 \mathrm{GeV}^{2}$. Such measurements will allow to quantify in detail the higher Fock components in the $N$ and $\Delta$ wave functions versus the simple three-quark Fock component, and pave the road to "asymptopia".

\section{OUTLOOK}

In summary, the impressive level of precision obtained in the measurements of the electromagnetic excitation of the $\Delta$-resonance during the past few years has challenged the theory to arrive at a QCD based description of the $\gamma N \Delta$ transition. Several new theoretical developments are under way and have shown promise and potential. We hope that this workshop will stimulate further efforts in this direction and contribute to the very fruitful interplay between theory and experiment in this field.

\section{ACKNOWLEDGMENTS}

I like to thank Aron Bernstein and Costas Papanicolas for organizing this stimulating workshop. I also like to thank Vladimir Pascalutsa for many fruitful collaborations on the topics discussed in this paper. This work is partially supported by DOE grant no. DE-FG02-04ER41302 and contract DE-AC05-06OR23177 under which Jefferson Science Associates operates the Jefferson Laboratory.

\section{REFERENCES}

1. D. Drechsel and L. Tiator, J. Phys. G 18, 449 (1992).

2. B. Krusche and S. Schadmand, Prog. Part. Nucl. Phys. 51, 399 (2003).

3. V. D. Burkert and T. S. H. Lee, Int. J. Mod. Phys. E 13, 1035 (2004).

4. V. Pascalutsa, M. Vanderhaeghen and S. N. Yang, Phys. Rept. (in press) [arXiv:hep-ph/0609004].

5. S. Weinberg, Physica A 96, 327 (1979).

6. J. Gasser and H. Leutwyler, Annals Phys. 158, 142 (1984); Nucl. Phys. B 250, 465 (1985).

7. V. Bernard, N. Kaiser, and U. G. Meißner, Int. J. Mod. Phys. E 4, 193 (1995).

8. H. F. Jones and M. D. Scadron, Ann. Phys. 81, 1 (1973).

9. L. Tiator, D. Drechsel, S. S. Kamalov and S. N. Yang, Eur. Phys. J. A 17, 357 (2003).

10. R. Beck et al., Phys. Rev. Lett. 78, 606 (1997).

11. R. Beck t al., Phys. Rev. C 61, 035204 (2000).

12. G. B lanpied et al., Phys. Rev. Lett. 79, 4337 (1997).

13. G. B lanpied et al., Phys. Rev. C 64, 025203 (2001).

14. W. M. Yao et al. [Particle Data Group], J. Phys. G 33, 1 (2006).

15. L. Tiator, D. Drechsel, O. Hanstein, S. S. Kamalov and S. N. Yang, Nucl. Phys. A 689, 205 (2001).

16. N. Isgur and G. Karl, Phys. Rev. D 18, 4187 (1978); ibid. D 19, 2653 (1979) [Erratum-ibid. D 23, 817 (1981)]; ibid. D 20 1191 (1979).

17. A. De Rujula, H. Georgi and S. L. Glashow, Phys. Rev. D 12, 147 (1975).

18. R. Koniuk and N. Isgur, Phys. Rev. D 21, 1868 (1980) [Erratum-ibid. D 23, 818 (1981)].

19. N. Isgur, G. Karl and R. Koniuk, Phys. Rev. D 25, 2394 (1982).

20. C. M. Becchi and G. Morpurgo, Phys. Lett. 17, 352 (1965).

21. J. F. Donoghue, E. Golowich and B. R. Holstein, Phys. Rev. D 12, 2875 (1975).

22. S. L. Glashow, Physica 96A, 27 (1979).

23. S. S. Gershtein and G. V. Jikia, Sov. J. Nucl. Phys. 34, 870 (1981) [Yad. Fiz. 34, 1566 (1981)].

24. M. Bourdeau and N. C. Mukhopadhyay, Phys. Rev. Lett. 58, 976 (1987).

25. S. A. Gogilidze, Yu. S. Surovtsev and F. G. Tkebuchava, Sov. J. Nucl. Phys. 45, 674 (1987) [Yad. Piz. 45, 1085 (1987)].

26. D. Drechsel and M. M. Giannini, Phys. Lett. B 143, 329 (1984).

27. M. M. Giannini, Rept. Prog. Phys. 54, 453 (1990).

28. S. Capstick and G. Karl, Phys. Rev. D 41, 2767 (1990).

29. S. Capstick, in Phys. Rev. D 46, 2864 (1992).

30. G. Kaelbermann and J. M. Eisenberg, Phys. Rev. D 28, 71 (1983).

31. A. W. Thomas, Adv. Nucl. Phys. 13, 1 (1984).

32. K. Bermuth, D. Drechsel, L. Tiator and J. B. Seaborn, Phys. Rev. D 37, 89 (1988). 
33. D. H. Lu, A. W. Thomas and A. G. Williams, Phys. Rev. C 55, 3108 (1997).

34. M. Fiolhais, B. Golli and S. Sirca, Phys. Lett. B 373, 229 (1996).

35. A. Wirzba and W. Weise, Phys. Lett. B 188, 6 (1987).

36. A. Abada, H. Weigel and H. Reinhardt, Phys. Lett. B 366, 26 (1996).

37. H. Walliser and G. Holzwarth, Z. Phys. A 357, 317 (1997).

38. D. I. Diakonov and V. Petrov, Nucl. Phys. B272, 457 (1986).

39. D. I. Diakonov, V. Petrov and P. Pobylitsa, Nucl. Phys. B306, 809 (1988).

40. T. Watabe, C. V. Christov and K. Goeke, Phys. Lett. B 349, 197 (1995).

41. A. Silva, D. Urbano, T. Watabe, M. Fiolhais and K. Goeke, Nucl. Phys. A 675, 637 (2000).

42. A. J. Buchmann, E. Hernandez and A. Faessler, Phys. Rev. C 55, 448 (1997).

43. A. J. Buchmann, these Proceedings.

44. A. J. Buchmann and E. M. Henley, Phys. Rev. C 63, 015202 (2001).

45. A. Bohr and B. Mottelson, Nuclear Structure II (Benjamin, Reading, MA, 1975).

46. A. Faessler, T. Gutsche, B. R. Holstein, V. E. Lyubovitskij, D. Nicmorus and K. Pumsa-ard, Phys. Rev. D 74, 074010 (2006).

47. G. 't Hooft, Nucl. Phys. B 72, 461 (1974).

48. E. Witten, Nucl. Phys. B 160, 57 (1979).

49. E. Jenkins, Ann. Rev. Nucl. Part. Sci. 48, 81 (1998).

50. R. F. Lebed, Czech. J. Phys. 49, 1273 (1999)

51. E. Jenkins and A. V. Manohar, Phys. Lett. B 335, 452 (1994).

52. E. Jenkins, X. d. Ji and A. V. Manohar, Phys. Rev. Lett. 89, 242001 (2002).

53. A. J. Buchmann, J. A. Hester and R. F. Lebed, Phys. Rev. D 66, 056002 (2002).

54. T. D. Cohen, Phys. Lett. B 554, 28 (2003).

55. T. D. Cohen and R. F. Lebed, Phys. Rev. D 74, 056006 (2006).

56. M. N. Butler, M. J. Savage and R. P. Springer, Phys. Lett. B 304, 353 (1993).

57. E. Jenkins and A. V. Manohar, Phys. Lett. B 255, 558 (1991).

58. G. C. Gellas, T. R. Hemmert, C. N. Ktorides and G. I. Poulis, Phys. Rev. D 60, 054022 (1999).

59. T. Hemmert, B. R. Holstein and J. Kambor, Phys. Lett. B 395, 89 (1997); J. Phys. G 24, 1831 (1998).

60. T. A. Gail and T. R. Hemmert, arXiv:nucl-th/0512082.

61. T. A. Gail and T. R. Hemmert, arXiv:nucl-th/0610081; these Proceedings.

62. V. Pascalutsa and M. Vanderhaeghen, Phys. Rev. Lett. 95, 232001 (2005).

63. V. Pascalutsa and M. Vanderhaeghen, Phys. Rev. D 73, 034003 (2006).

64. V. Pascalutsa and M. Vanderhaeghen, arXiv:hep-ph/0611317; these Proceedings.

65. V. Pascalutsa and D. R. Phillips, Phys. Rev. C 67, 055202 (2003).

66. D. Elsner et al., Eur. Phys. J. A 27, 91 (2006).

67. T. Pospischil et al., Phys. Rev. Lett. 86, 2959 (2001).

68. S. Stave et al., arXiv:nucl-ex/0604013.

69. N. F. Sparveris et al. [OOPS Collaboration], Phys. Rev. Lett. 94, 022003 (2005).

70. K. Joo et al. [CLAS Collaboration], Phys. Rev. Lett. 88, 122001 (2002).

71. C. Alexandrou, P. de Forcrand, H. Neff, J. W. Negele, W. Schroers and A. Tsapalis, Phys. Rev. Lett. 94,021601 (2005).

72. T. Sato and T.-S.H. Lee, Phys. Rev. C 54, 2660 (1996); ibid. 63, 055201 (2001)

73. S. S. Kamalov and S. N. Yang, Phys. Rev. Lett. 83, 4494 (1999);

S. S. Kamalov, S. N. Yang, D. Drechsel, O. Hanstein, and L. Tiator, Phys. Rev. C 64, 032201(R) (2001).

74. D. Drechsel, O. Hanstein, S. S. Kamalov and L. Tiator, Nucl. Phys. A 645, 145 (1999).

75. R. A. Arndt, W. J. Briscoe, I. I. Strakovsky and R. L. Workman, Phys. Rev. C 66, 055213 (2002).

76. M. De Sanctis, M. M. Giannini, E. Santopinto and A. Vassallo, Nucl. Phys. A 755, 294 (2005).

77. D. B. Leinweber, T. Draper and R. M. Woloshyn, Phys. Rev. D 48, 2230 (1993).

78. C. Alexandrou, P. de Forcrand and A. Tsapalis, Phys. Rev. D 66, 094503 (2002).

79. C. Alexandrou, arXiv:hep-lat/0608025; these Proceedings.

80. C. Alexandrou et al., Phys. Rev. D 69, 114506 (2004).

81. C. Mertz et al., Phys. Rev. Lett. 86, 2963 (2001).

82. D. B. Leinweber, A. W. Thomas and R. D. Young, Phys. Rev. Lett. 86, 5011 (2001);

W. Detmold, W. Melnitchouk, J. W. Negele, D. B. Renner and A. W. Thomas, ibid. 87, 172001 (2001).

83. T. R. Hemmert, M. Procura and W. Weise, Phys. Rev. D 68, 075009 (2003).

84. M. K. Banerjee and J. Milana, Phys. Rev. D 52, 6451 (1995).

85. D. B. Leinweber, A. W. Thomas, K. Tsushima and S. V. Wright, Phys. Rev. D 61, 074502 (2000).

86. R. D. Young, D. B. Leinweber, A. W. Thomas and S. V. Wright, Phys. Rev. D 66, 094507 (2002);

R. D. Young, D. B. Leinweber and A. W. Thomas, Prog. Part. Nucl. Phys. 50, 399 (2003);

D. B. Leinweber, A. W. Thomas and R. D. Young, Phys. Rev. Lett. 92, 242002 (2004).

87. V. Bernard, T. R. Hemmert and U. G. Meissner, Phys. Lett. B 565, 137 (2003).

88. M. Frink, U. G. Meissner and I. Scheller, Eur. Phys. J. A 24, 395 (2005).

89. V. Bernard, T. R. Hemmert and U. G. Meissner, Phys. Lett. B 622, 141 (2005).

90. C. Hacker, N. Wies, J. Gegelia and S. Scherer, Phys. Rev. C 72, 055203 (2005)

91. V. Pascalutsa and M. Vanderhaeghen, Phys. Lett. B 636, 31 (2006). 
92. J. N. Labrenz and S. R. Sharpe, Phys. Rev. D 54, 4595 (1996).

93. C. W. Bernard et al., Phys. Rev. D 64, 054506 (2001).

94. R. D. Young, D. B. Leinweber and A. W. Thomas, Nucl. Phys. Proc. Suppl. 129, 290 (2004).

95. V. Pascalutsa and M. Vanderhaeghen, Phys. Rev. Lett. 94, 102003 (2005).

96. D. Muller, D. Robaschik, B. Geyer, F. M. Dittes and J. Horejsi, Fortsch. Phys. 42, 101 (1994)

97. X. D. Ji, Phys. Rev. Lett. 78, 610 (1997); Phys. Rev. D 55, 7114 (1997).

98. A. V. Radyushkin, Phys. Lett. B 380, 417 (1996).

99. X. D. Ji, J. Phys. G 24, 1181 (1998).

100. K. Goeke, M. V. Polyakov and M. Vanderhaeghen, Prog. Part. Nucl. Phys. 47, 401 (2001).

101. M. Diehl, Phys. Rept. 388, 41 (2003).

102. A. V. Belitsky and A. V. Radyushkin, Phys. Rept. 418, 1 (2005).

103. P. A. M. Guichon, L. Mosse and M. Vanderhaeghen, Phys. Rev. D 68, 034018 (2003).

104. M. Guidal et al. Nucl. Phys. A 721, 327 (2003).

105. L. L. Frankfurt, M. V. Polyakov, M. Strikman and M. Vanderhaeghen, Phys. Rev. Lett. 84, 2589 (2000).

106. P. Stoler, Phys. Rev. Lett. 91, 172303 (2003); Phys. Rev. D 65, 053013 (2002).

107. M. Diehl, T. Feldmann, R. Jakob and P. Kroll, Eur. Phys. J. C 39, 1 (2005)

108. M. Guidal, M. V. Polyakov, A. V. Radyushkin and M. Vanderhaeghen, Phys. Rev. D 72, 054013 (2005).

109. P. Kroll, these Proceedings.

110. V. Pascalutsa and M. Vanderhaeghen, arXiv:hep-ph/0611050.

111. V. V. Frolov et al., Phys. Rev. Lett. 82, 45 (1999)

112. M. Ungaro, P. Stoler, I. Aznauryan, V. D. Burkert, K. Joo and L. C. Smith [CLAS Collaboration], Phys. Rev. Lett. 97,112003 (2006).

113. N. F. Sparveris et al., arXiv:nucl-ex/0611033; N. F. Sparveris, these Proceedings.

114. J.J. Kelly et al., Phys. Rev. Lett. 95, 102001 (2005).

115. M. Burkardt, Phys. Rev. D 62, 071503 (2000) [Erratum-ibid. D 66, 119903 (2002)].

116. A. V. Belitsky, X. d. Ji and F. Yuan, Phys. Rev. D 69, 074014 (2004).

117. M. Burkardt, Int. J. Mod. Phys. A 18, 173 (2003).

118. G. P. Lepage and S. J. Brodsky, Phys. Rev. D 22, 2157 (1980).

119. V. L. Chernyak and A. R. Zhitnitsky, JETP Lett. 25, 510 (1977) [Pisma Zh. Eksp. Teor. Fiz. 25, 544 (1977)].

120. V. L. Chernyak, A. R. Zhitnitsky and V. G. Serbo, JETP Lett. 26, 594 (1977) [Pisma Zh. Eksp. Teor. Fiz. 26, 760 (1977)].

121. A. V. Efremov and A. V. Radyushkin, Phys. Lett. B 94, 245 (1980).

122. C. E. Carlson, Phys. Rev. D 34, 2704 (1986).

123. A. F. Sill et al., Phys. Rev. D 48, 29 (1993).

124. M. K. Jones et al. [Jefferson Lab Hall A Collaboration], Phys. Rev. Lett. 84 1398 (2000).

125. V. Punjabi et al., Phys. Rev. C 71, 055202 (2005) [Erratum-ibid. C 71, 069902 (2005)].

126. O. Gayou et al., Phys. Rev. C 64, 038202 (2001).

127. O. Gayou et al. [Jefferson Lab Hall A Collaboration], Phys. Rev. Lett. 88, 092301 (2002).

128. A. V. Belitsky, X. d. Ji and F. Yuan, Phys. Rev. Lett. 91, 092003 (2003).

129. A. Idilbi, X. d. Ji and J. P. Ma, Phys. Rev. D 69, 014006 (2004).

130. V. M. Belyaev and A. V. Radyushkin, Phys. Lett. B 359, 194 (1995).

131. V. M. Belyaev and A. V. Radyushkin, Phys. Rev. D 53, 6509 (1996).

132. R. P. Feynman, Photon-Hadron Interactions (Benjamin, Reading, MA, 1972). 